\title{
The Thermodynamic Limit for a Crystal
}

\author{
C. Fefferman ${ }^{\star}$ \\ Department of Mathematics, Princeton University, Princeton, New Jersey 08544, USA
}

\begin{abstract}
Consider a crystal with nucleii fixed at the lattice points in $\Omega \subset \mathbb{R}^{3}$, interacting by Coulomb forces with quantized electrons in $\Omega$. We prove that the pressure tends to a limit as $\Omega$ grows infinitely large.
\end{abstract}

\section{Introduction}

A natural model for electrons in a crystal is as follows. We place a nucleus of charge +1 at each lattice point in a box $\Omega \subseteq \mathrm{R}^{3}$. The basic Hamiltonian for $N$ quantized electrons $x_{1}, \ldots, x_{N}$ in $\Omega$ is

$$
H_{N, \Omega}=-\Delta_{x}+\sum_{j<k}\left|x_{j}-x_{k}\right|^{-1}+\sum_{j<k}\left|y_{j}-y_{k}\right|^{-1}-\sum_{j, k}\left|x_{j}-y_{k}\right|^{-1}
$$

with Dirichlet boundary conditions on $\Omega \times \cdots \times \Omega$. Here $y_{1} \ldots y_{M}$ are the nucleii, and $H_{N, \Omega}$ acts on antisymmetric wave functions $\psi\left(x_{1} \ldots x_{N}\right)$. If the electrons have temperature $\beta^{-1}$ and chemical potential $\mu / \beta$, then up to trivial factors the pressure is given by

$$
F=(\operatorname{Vol} \Omega)^{-1} \ln \left[\sum_{N} e^{\mu N} \operatorname{Trace} e^{-\beta H_{N, \Omega}}\right] .
$$

The purpose of this paper is to prove that $F$ tends to a limit as the volume of $\Omega$ tends to infinity. This is called existence of the thermodynamic limit. See Sect. 2 for the precise statement of our result. The problem of the thermodynamic limit for crystals was posed by Lebowitz and Lieb, following their basic work [1] on real matter, with electrons and nucleii all quantized. Since a crystal is not rotationally symmetric, the method of [1] doesn't work here.

Of course one wants to allow periodic arrangements of nucleii more general than just charge +1 at each lattice point; also, we should introduce spin into our wave functions. These refinements can be easily incorporated into our proof. For that matter, it is enough to suppose that the placement of nucleii is asymptotically periodic; and our electrons could be Bosons (or even classical particles provided the nucleii have hard cores).

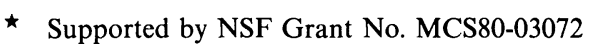


In a later article, we shall apply our technique to show that quantized electrons and nucleii at suitable temperature and density form an ideal gas of hydrogen atoms or molecules.

\section{Notation}

Let $\Gamma=\left\{\Omega \subset R^{3} \mid \Omega\right.$ bounded, convex, with non-empty interior $\}$,

$\Gamma_{0}=\{D \in \Gamma \mid \partial D$ is smooth and has strictly positive Gaussian curvature at every point $\}$.

For $D \in \Gamma, x \in R^{3}, R>0$, write $D(x, R)$ for the translate and dilate, $\{R y+x \mid y \in D\}$. We write $Q^{0}$ for the unit cube in $R^{3}$.

Set $L_{N}^{2}(\Omega)=\left\{\right.$ square-integrable antisymmetric $\psi\left(x_{1} \ldots x_{N}\right)$ on $\left.\Omega^{N}\right\}$,

$$
L_{*}^{2}(\Omega)=\sum_{N \geqq 0} \oplus L_{N}^{2}(\Omega) \text {. }
$$

If $\psi \in L_{N}^{2}(\Omega)$ and $\left(x_{1} \ldots x_{N}\right) \notin \Omega^{N}$, then we interpret $\psi\left(x_{1} \ldots x_{N}\right)$ to be zero.

If $K(x)$ is a function on $R^{3}$, and we have electrons $x_{1} \ldots x_{N}$ and nucleii $y_{1} \ldots y_{M}$, then $V[K]=\frac{1}{2} \sum_{j \neq k} K\left(x_{j}-x_{k}\right)+\frac{1}{2} \sum_{j \neq k} K\left(y_{j}-y_{k}\right)-\sum_{j, k} K\left(x_{j}-y_{k}\right)$.

Thus, the Coulomb potential is $V\left[|x|^{-1}\right]$.

For $\Omega \subset R^{3}$, define $H_{N, \Omega}^{0}=-\Delta$ on $L_{N}^{2}(\Omega)$ with Dirichlet boundary conditions; $H_{N, \Omega}=-\Delta+V\left[|x|^{-1}\right]$ on $L_{N}^{2}(\Omega)$ with Dirichlet boundary conditions, where the nucleii are placed at all the points of $Z^{3} \cap \Omega$.

$$
\text { Define } F(\mu, \beta, \Omega)=|\Omega|^{-1} \ln \left[\sum_{N \geqq 0} e^{\mu N} \operatorname{Tr} e^{-\beta H_{N, \Omega}}\right] \text {. }
$$

If $\Omega=D(x, R)$ for $D \in \Gamma$, then we write $F(\mu, \beta, x, R, D)$ for $F(\mu, \beta, \Omega)$. Observe that $F$ is invariant under translates of $x$ by vectors in $Z^{3}$, but not by vectors in $R^{3}$.

When $\mu, \beta, D$ are kept fixed, we shall often write $F(x, R)$ for $F(\mu, \beta, x, R, D)$.

\section{Reduction of the Theorem to Two Main Lemmas}

The precise statement of our result is as follows.

Theorem. For each $\beta>0, \mu \in R^{1}, \Omega \in \Gamma$, the limit $\lim _{R \rightarrow \infty} F(\mu, \beta, x, R, \Omega)$ converges uniformly in $x$. Its value is independent of $\Omega$ and has the form $\phi(\beta)+\mu$.

In this section, we shall state two main lemmas, and show how they imply the theorem. The rest of the paper is devoted to proving the lemmas.

Lemma 1. Let $D \in \Gamma_{0}, \Omega \in \Gamma, \varepsilon>0$. Suppose we have radii $R_{1}<R_{2}<\cdots<R_{M}<R_{*}$ with $R_{1}>C \varepsilon^{-10}, R_{k+1}>2 R_{k}, M>C \varepsilon^{-10}$, and $R_{*}>M^{10} R_{M}$. Then for $x \in R^{3}$ we have

$$
F\left(\mu, \beta, x, R_{*}, \Omega\right)<\varepsilon+\max _{1 \leqq k \leqq M}\left[\mathrm{Av}_{y \in Q^{0}} F\left(\mu, \beta, y, R_{k}, D\right)\right] .
$$

The constants $C$ in Lemma 1 depend on $\mu, \beta, D, \Omega$. 
Lemma 2. For $\Omega \in \Gamma$ there is a constant $C(\Omega)$ with the following property. Let $D \in \Gamma_{0}$, $0<\varepsilon<1, D_{*}=D\left(x^{\prime}, R^{\prime}\right), \Omega_{*}=\Omega(x, R)$. Suppose $D_{*} \subseteq \Omega_{*}$, dist $\left(\partial D_{*}, \partial \Omega_{*}\right)>10$, and $\left|\Omega_{*}\right|<\left(1+\varepsilon^{10}\right)\left|D_{*}\right|$. Then $F\left(\mu, \beta, \Omega_{*}\right)>F\left(\mu, \beta, D_{*}\right)-C(\Omega) \varepsilon$ if $R^{\prime}$ is sufficiently large.

Very roughly, Lemma 1 says that $F(\mu, \beta, \Omega)$ is monotone decreasing in $\Omega$ over the long run, while Lemma 2 says that a small increase in $\Omega$ will not cause a large drop in $F(\mu, \beta, \Omega)$.

Let us check that Lemmas 1 and 2 imply the theorem. From Lemma 1 we get

Corollary 1. For fixed $\mu, \beta, D \in \Gamma_{0}$, the quantity $\bar{F}(R) \equiv A V_{y \in Q^{\circ}} F(\mu, \beta, y, R, D)$ tends to a limit as $R \rightarrow \infty$.

Proof. Let $l=\liminf _{R \rightarrow \infty} \bar{F}(R)$, and take $\varepsilon>0$. It is trivial to show $l \neq-\infty$ (see estimate (3.20) below), so there are arbitrarily large $R$ with $\bar{F}(R) \leqq l+\varepsilon$. So we can pick successively $R_{1}, R_{2}, \ldots, R_{M}$ to satisfy $R_{1}>C \varepsilon^{-10}, R_{k+1}>2 R_{k}, M>C \varepsilon^{-10}$, and $\bar{F}\left(R_{k}\right) \leqq l+\varepsilon$. Here the constant $C$ is taken from Lemma 1 with $\Omega=D$. Lemma 1 gives $F\left(\mu, \beta, x, R_{*}, D\right) \leqq \varepsilon+[l+\varepsilon]$ for all $x \in R^{3}, R_{*}>M^{10} R_{M}$. Averaging over $x \in Q^{0}$, we get $\bar{F}\left(R_{*}\right) \leqq l+2 \varepsilon$ for $R_{*}$ large enough, so $\limsup _{R \rightarrow \infty} \bar{F}(R) \leqq 2 \varepsilon+l=$ $2 \varepsilon+\liminf _{R \rightarrow \infty} \bar{F}(R)$.

Q.E.D.

Now let $\bar{F}(\mu, \beta, D)=\lim _{R \rightarrow \infty} A v_{y \in Q^{\circ}} F(\mu, \beta, y, R, D)$. Corollary 1 and Lemma 1 together show at once

Corollary 2. Given $\mu, \beta, D \in \Gamma_{0}, \Omega \in \Gamma$ and $\varepsilon>0$, we have $F(\mu, \beta, x, R, \Omega) \leqq$ $\varepsilon+\bar{F}(\mu, \beta, D)$ if $R$ is large enough.

In particular,

$$
F(\mu, \beta, x, R, D) \leqq \varepsilon+\bar{F}(\mu, \beta, D) \quad \text { if } R \text { is large enough. }
$$

On the other hand, Lemma 2 shows that for a large constant $C$ we have $F(\mu, \beta, x, R, D) \geqq F(\mu, \beta, y, R-C, D)-C(D) \varepsilon$ if $R$ is large enough and $|x-y|<50$. Average this estimate over all $y$ in a translate of $Q^{0}$ containing $x$. The result is $F(\mu, \beta, x, R, D) \geqq\left[\mathrm{Av}_{y \in Q^{0}} F(\mu, \beta, y, R-C, D)\right]-C(D) \varepsilon$ for large $R$. Recalling the definition of $\bar{F}(\mu, \beta, D)$, we conclude that

$$
F(\mu, \beta, x, R, D) \geqq \bar{F}(\mu, \beta, D)-C^{\prime}(D) \varepsilon \quad \text { if } R \text { is large enough. }
$$

Comparing with (2.2), we find that $F(\mu, \beta, x, R, D) \rightarrow \bar{F}(\mu, \beta, D)$ as $R \rightarrow \infty$, for each $D \in \Gamma_{0}$.

Next note that $\bar{F}(\mu, \beta, D)$ is independent of $D$. This is immediate from Corollary 2 with $\Omega \in \Gamma_{0}$. We write $\bar{F}(\mu, \beta)$ for $\bar{F}(\mu, \beta, D)$.

Finally, let $\Omega \in \Gamma, \varepsilon>0$, and pick $D_{\varepsilon} \in \Gamma_{0}$ so that $\bar{D}_{\varepsilon} \subseteq$ interior $\Omega,|\Omega|<$ $\left(1+\varepsilon^{10}\right)\left|D_{\varepsilon}\right|$. Lemma 2 shows that $F(\mu, \beta, x, R, \Omega)>F\left(\mu, \beta, x, R, D_{\varepsilon}\right)-C(\Omega) \varepsilon$ for $R$ large enough. Hence $F(\mu, \beta, x, R, \Omega)>\bar{F}(\mu, \beta)-2 C(\Omega) \varepsilon$ for large $R$. On the other hand, Corollary 2 with $D=D_{\varepsilon}$ gives $F(\mu, \beta, x, R, \Omega) \leqq \bar{F}(\mu, \beta)+\varepsilon$ for large $R$. So $\lim _{R \rightarrow \infty} F(\mu, \beta, R, \Omega)=\bar{F}(\mu, \beta)$ uniformly in $x$, for any $\Omega \in \Gamma$. Our theorem is completely proved, except for the assertion $\bar{F}(\mu, \beta)=\mu+\phi(\beta)$, which follows trivially from estimate (3.6) below. Hence, the problem is reduced to proving Lemmas 1 and 2. 


\section{Estimates for Coulomb Systems}

Consider a Coulomb system with electrons $x_{1} \ldots x_{N}$ and nucleii $\omega_{1} \ldots \omega_{N^{\prime}}$. Assume $\left|\omega_{k}-\omega_{k^{\prime}}\right| \geqq 1$ for $k \neq k^{\prime}$. We shall compare the potential energy $V=V\left[|x|^{-1}\right]$ with the energy of a continuous charge distribution:

$$
\begin{gathered}
V_{\rho}=\frac{1}{2} \iint \frac{\rho(x) \rho(y)}{|x-y|} d x d y, \quad \rho(x)=\sum_{k} \phi\left(x-\omega_{k}\right)-\sum_{j} \phi\left(x-x_{j}\right), \\
\phi \in C_{0}^{\infty}(|x| \leqq 1 / 4), \quad \int \phi=1, \quad \phi \geqq 0 .
\end{gathered}
$$

First of all, $V_{\rho}$ contains $N$ "self-energy" terms $\frac{1}{2} \int \phi\left(x-x_{j}\right) \phi\left(y-x_{k}\right) /|x-y| d x d y$ with $j=k$, as well as $N^{\prime}$ similar terms for the nucleii. These terms have no analogues in $V\left[|x|^{-1}\right]$; they total $C N+C N^{\prime}$. have

Next, compare the terms in $V, V_{\rho}$ arising from repulsion of distinct electrons. We

$$
\left|x_{j}-x_{k}\right|^{-1} \geqq c\left|x_{j}-x_{k}\right|^{-1} \chi_{\left|x_{j}-x_{k}\right|<1 / 10}+\int \frac{\phi\left(x-x_{j}\right) \phi\left(y-x_{k}\right)}{|x-y|} d x d y .
$$

in view of the subharmonicity of the Coulomb potential. ${ }^{1}$

The terms in $V, V_{\rho}$ arising from repulsion of distinct nucleii are exactly equal, since distinct nucleii are at least distance 1 apart. Finally, the electron-proton attraction gives rise to terms in $V, V_{\rho}$ which compare as follows:

$$
-\left|x_{j}-\omega_{k}\right|^{-1} \geqq-\int \frac{\phi\left(x-x_{j}\right) \phi\left(y-\omega_{k}\right)}{|x-y|} d x d y-\left|x_{j}-\omega_{k}\right|^{-1} \chi_{\left|x_{j}-\omega_{k}\right|<1 / 2} .
$$

Consequently,

$$
\begin{aligned}
V\left[|x|^{-1}\right] \geqq & V_{\rho}+c \sum_{0<\left|x_{j}-x_{k}\right|<1 / 10}\left|x_{j}-x_{k}\right|^{-1} \\
& -\sum_{\left|x_{j}-\omega_{k}\right|<1 / 2}\left|x_{j}-\omega_{k}\right|^{-1}-C N-C N^{\prime} .
\end{aligned}
$$

For functions $\psi$ of three variables, we have an elementary inequality

$$
\int_{|x-\omega|<1 / 2}^{\frac{1}{2}}\left|\nabla_{x} \psi\right|^{2} d x \geqq 2 \int_{|x-\omega|<1 / 2}|x-\omega|^{-1}|\psi(x)|^{2} d x-C \int_{|x-\omega|<1 / 2}|\psi(x)|^{2} d x .
$$

This amounts to the stability of a single hydrogen atom. Writing $x_{j}$ for $x, \omega_{k}$ for $\omega$; integrating against $\prod_{l \neq j} d x_{l}$; and summing over $j, k$ we obtain

$$
-\frac{1}{2} \Delta \geqq 2 \sum_{\left|x_{j}-\omega_{k}\right|<1 / 2}\left|x_{j}-\omega_{k}\right|^{-1}-C N-C N^{\prime}
$$

as operators on $\psi\left(x_{1} \ldots x_{N}\right)$. Adding (3.1) and (3.2), we get for $H=-\Delta+V\left[|x|^{-1}\right]$ the operator inequality

$$
\begin{aligned}
H+C N+C N^{\prime} \geqq & -\frac{1}{2} \Delta+c \sum_{0<\left|x_{j}-x_{k}\right|<1 / 10}\left|x_{j}-x_{k}\right|^{-1} \\
& +\sum_{\left|x_{j}-\omega_{k}\right|<1 / 2}\left|x_{j}-\omega_{k}\right|^{-1}+V_{\rho} .
\end{aligned}
$$

1 Here we assume $\phi(x) \geqq c$ when $|x|<1 / 10$ 
The terms on the right are all positive, so (3.3) implies $H$-stability of the system. Note that we did not need antisymmetric wave functions.

Lemma 3. Let $F$ be a function of compact support on $R^{3}$, with one distributional derivative in $L^{2}$. Then

$$
\left|\sum_{k} \phi * F\left(\omega_{k}\right)-\sum_{j} \phi * F\left(x_{j}\right)\right|^{2} \leqq C\|\nabla F\|_{L^{2}}^{2} \cdot\left(H+C N+C N^{\prime}\right)
$$

as operators on $L^{2}\left(R^{3 N}\right)$.

Corollary. If the system is confined to a ball of radius $R$, then the net charge $N-N^{\prime}$ satisfies

$$
\left(N-N^{\prime}\right)^{2} \leqq C R\left(H+C N+C N^{\prime}\right)
$$

In particular, for nucleii at the lattice points of $\Omega(x, R)$, we have

$$
H \geqq-\frac{1}{2} \Delta+c \delta^{2} R^{5}
$$

if the net charge $\left|N-N^{\prime}\right|>\delta R^{3}, \delta>C R^{-1}$.

Proof of the Corollary. Estimate (3.5) is just the special case of Lemma 3 with $F(x)=1$ for $x$ in a ball of radius $2 R, F(x)=0$ outside a ball of radius $3 R$, $|\nabla F| \leqq C R^{-1}$ everywhere. To prove (3.6), note that $N^{\prime} \sim(\operatorname{Vol} \Omega) \cdot R^{3}$, so if $\left|N-N^{\prime}\right|>\delta R^{3}$ with $\delta>C R^{-1}$ then (3.5) shows that $H \geqq c \delta^{2} R^{5}+C_{1} N$, while (3.3) gives $H \geqq-\frac{1}{2} \Delta-C_{1} N-C R^{3}$. Estimate (3.6) follows by adding the last two inequalities.

Proof of Lemma 3. We have $\sum_{k} \phi * F\left(\omega_{k}\right)-\sum_{k} \phi * F\left(x_{j}\right)=\langle\rho, F\rangle=\left\langle(-\Delta)^{-1 / 2} \rho\right.$, $\left.(-\Delta)^{1 / 2} F\right\rangle$. The formal manipulation is justified if $F \in C_{0}^{\infty}$, which we may assume. Thus,

$$
\begin{aligned}
\left|\sum_{k} \phi * F\left(\omega_{k}\right)-\sum_{j} \phi * F\left(x_{j}\right)\right|^{2} & \leqq\left\|(-\Delta)^{-1 / 2} \rho\right\|^{2} \cdot\left\|(-\Delta)^{1 / 2} F\right\|^{2}=\|\nabla F\|^{2}\left\langle(-\Delta)^{-1} \rho, \rho\right\rangle \\
& =\text { const }\|\nabla F\|^{2} V_{\rho} .
\end{aligned}
$$

So (3.4) follows from (3.3).

Next we give an estimate for $V[K]$ when $K$ behaves roughly like $|x|^{-1}$ in the following rather technical sense.

$$
\begin{aligned}
\left|\partial_{x}^{\alpha} K(x)\right| \leqq C|x|^{-1-|\alpha|} & \text { for }|\alpha| \leqq 2 \text { and all } x . \\
\left|\partial_{x}^{\alpha} K(x)\right| \leqq C|x|^{-4} & \text { for }|\alpha|=3,
\end{aligned}
$$

unless $x$ belongs to one of the annuli $\mathscr{A}_{k}=D\left(0, R_{k}+1\right) \backslash D\left(0, R_{k}-1\right)$. Here we assume $D \in \Gamma_{0}$ and $R_{1}, R_{1}, \ldots$ are fixed radii satisfying $R_{1} \geqq 10, R_{k+1} \geqq 2 R_{k}$.

Lemma 4. If $K$ satisfies (3.7) and (3.8), then $V[K] \leqq C\left(H+C N+C N^{\prime}\right)$.

Proof. First we check that (3.7), (3.8), imply a bound for the Fourier transform of $K$, namely $|\widehat{R}(\xi)| \leqq C|\xi|^{-2}$. In fact, we can write $K=K_{1}+K_{2}$ with $K_{1}$ supported in $|x|$ $<2|\xi|^{-1}, K_{2}$ supported in $|x|>|\xi|^{-1}$, and $K_{1}, K_{2}$ satisfying (3.7) and (3.8). Then one 
checks that $\left\|K_{1}\right\|_{L^{1}} \leqq C|\xi|^{-2}$ and $\int\left|\Delta K_{2}(x)-\Delta K_{2}(x-y)\right| d x \leqq C$ for $|y|<c|\xi|^{-1}$. Consequently $\left|\widehat{K}_{1}(\xi)\right| \leqq C|\xi|^{-2}$, while $\left.\left|\left[1-e^{i v \cdot \xi}\right]\right| \xi\right|^{2} \widehat{K}_{2}(\xi) \mid \leqq C$ for $|y|<c|\xi|^{-1}$. Taking $y=(c / 2) \xi|\xi|^{-2}$, we get $\left|\widehat{K}_{2}(\xi)\right| \leqq C^{\prime}|\xi|^{-2}$, and so $|\widehat{K}(\xi)| \leqq C|\xi|^{-2}$ as claimed.

Now set $K^{\#}=|x|^{-1}-c K(x)$ with $0<c \ll 1$. We know that $K^{\#}$ has positive Fourier transform, so that $\int K^{\#}(x-y) \rho(x) \rho(y) d x d y \equiv K^{\#}\{\rho\} \geqq 0$ for continuous charge distribution $\rho$.

We shall prove that

$$
V\left[K^{\sharp}\right] \geqq-C\left(H+C N+C N^{\prime}\right) .
$$

If (3.9) holds, then since $V[1 /|x|] \leqq H$ and $V[c K-1 /|x|] \leqq C\left(H+C N+C N^{\prime}\right)$, we obtain the conclusion of Lemma 4 just by adding. So the problem reduces to proving (3.9).

Subdivide $R^{3}$ into a grid $\left\{Q_{v}\right\}$ of cubes of side $10^{-3}$, and let $N_{v}$ be the number of particles $\left(x_{j}\right.$ and $\left.\omega_{k}\right)$ in $Q_{v}$. Evidently

$$
\begin{aligned}
\frac{1}{2} \sum_{v} N_{v}\left(N_{v}-1\right) \leqq & \sum_{0<\mid x_{j}-x_{k \mid}<10^{-2}}\left|x_{j}-x_{k}\right|^{-1} \\
& +\sum_{\left|x_{j}-\omega_{k}\right|<1 / 2}\left|x_{j}-\omega_{k}\right|^{-1} \leqq C\left(H+C N+C N^{\prime}\right)
\end{aligned}
$$

by (3.3). Therefore

$$
\sum_{v} N_{v}^{2} \leqq C\left(H+C N+C N^{\prime}\right) \text { since } \sum_{v} N_{v}=N+N^{\prime} .
$$

Now we are ready to prove (3.9) by imitating the proof of (3.3). Fix an even approximate identity $\psi \in C_{0}^{\infty}\left(|x|<10^{-3}\right)$ with $\int x^{\alpha} \psi(x) d x=\delta_{\alpha 0},|\alpha|<10$. Then set $\rho^{\#}(x)=\sum_{k} \psi\left(x-\omega_{k}\right)-\sum_{j} \psi\left(x-x_{j}\right)$, and compare $V\left[K^{\#}\right]$ with the non-negative quantity $\quad V^{\#}=\frac{1}{2} \int K^{\#}(x-y) \rho^{\#}(x) \rho^{\#}(y) d x d y$. As before, $V^{\#}$ contains selfenergy terms which total $C N+C N^{\prime}$. The difference between $K^{\#}\left(y_{1}-y_{2}\right)$ and the corresponding term $\int K^{\#}(x-y) \psi\left(x-y_{1}\right) \psi\left(y-y_{2}\right) d x d y$ is $\varepsilon\left(y_{1}-y_{2}\right)$ with $\varepsilon=K^{\#}-K^{\#} * \psi * \psi$. Therefore

$$
V\left[K^{\#}\right] \geqq V^{\#}-C N-C N^{\prime}-\sum_{y_{j} \neq y_{k}}\left|\varepsilon\left(y_{j}-y_{k}\right)\right|,
$$

where $y_{1} \ldots y_{N+N^{\prime}}$ is a list of all the particles $x_{1} \ldots x_{N}, \omega_{1} \ldots \omega_{N^{\prime}}$.

In view of the moment conditions on $\psi$, we have the estimates

$$
\begin{aligned}
& \left.\varepsilon(y)|\leqq C| y\right|^{-1} \text { just from the size of } K^{\#}, \\
& |\varepsilon(y)| \leqq C|y|^{-3} \text { by Taylor-expanding } K^{\#} \text { to first order using (3.7), } \\
& |\varepsilon(y)| \leqq C|y|^{-4} \text { outside } \bigcup_{k=1}^{\infty}\left[D\left(0, R_{k}+2\right) \backslash D\left(0, R_{k}-2\right)\right],
\end{aligned}
$$

by Taylor-expanding $K^{\#}$ to second order using (3.8).

$$
\text { Set } \varepsilon\left(Q_{\mu}, Q_{v}\right)=\max \left\{\left|\varepsilon\left(y-y^{\prime}\right)\right| \quad\left|y \in Q_{\mu}, y^{\prime} \in Q_{v},\right| y-y^{\prime} \mid>10^{-3}\right\} .
$$

From (3.13), (3.14) we get

$$
\sum_{v} \varepsilon\left(Q_{\mu}, Q_{v}\right)<C \text { for each } \mu ; \sum_{\mu} \varepsilon\left(Q_{\mu}, Q_{v}\right)<C \text { for each } v .
$$


On the other hand (3.11) and (3.12) imply

$$
V\left[K^{\#}\right] \geqq-C N-C N^{\prime}-\sum_{\mu, v} \varepsilon\left(Q_{\mu}, Q_{v}\right) N_{\mu} N_{v}-\sum_{0<\left|y_{j}-y_{k}\right|<10^{-2}} C\left|y_{j}-y_{k}\right|^{-1} .
$$

Now (3.15) shows that $\sum_{\mu, v} \varepsilon\left(Q_{\mu}, Q_{v}\right) N_{\mu} N_{v} \leqq C \sum_{\mu} N_{\mu}^{2}$, which we estimate by (3.10). Also

$$
\sum_{0<\left|y_{j}-y_{k}\right|<10^{-3}}\left|y_{j}-y_{k}\right|^{-1}=\sum_{0<\left|x_{j}-x_{k}\right|<10^{-3}}\left|x_{j}-x_{k}\right|^{-1}+\sum_{\left|x_{j}-\omega_{k}\right|<10^{-3}}\left|x_{j}-\omega_{k}\right|^{-1},
$$

which we estimate by (3.3). Hence (3.16) yields $V\left[K^{\#}\right] \geqq-C\left(H+C N+C N^{\prime}\right)$, which is the desired estimate (3.9).

We shall also need estimate on $\sum_{y \in J\left|x_{j}-y\right|<1 / 2}\left|x_{j}-y\right|^{-1}$ for various subsets $J \subseteq Z^{3}$. Imitating the proof of (3.2), we first note that $\varepsilon^{2} \int_{|x-y|<1 / 2}\left|\nabla_{x} \psi\right|^{2} d x \geqq$ $\int_{|x-y|<1 / 2}|x-y|^{-1}|\psi|^{2} d x-C(\varepsilon) \int_{|x-y|<1 / 2}|\psi|^{2} d x$, for functions $\stackrel{|x-y|<1 / 2}{\psi}$ on $R^{3}$ and $\varepsilon>0$ arbitrary. Set $x=x_{j}$, integrate against $\prod_{l \neq j} d x_{l}$, sum over $j$ and sum over $y \in J$. We obtain

$$
-\varepsilon^{2} \Delta \geqq \sum_{y \in J} \sum_{\left|x_{j}-y\right|<1 / 2}\left|x_{j}-y\right|^{-1}-C(\varepsilon) \cdot\left[\text { Number of } x_{j} \in \bigcup_{y \in J} B(y, 1 / 2)\right]
$$

as operators on $L^{2}\left(R^{3 N}\right)$. Setting $\mathfrak{N}=\left\{v \mid Q_{v}\right.$ meets one of the $\left.B(y, 1 / 2), y \in J\right\}$, we note that $|\mathfrak{N}| \leqq C|J|$, while the number of $x_{j} \in \bigcup_{y \in J} B(y, 1 / 2)$ is at most

$$
\begin{aligned}
\sum_{v \in \mathfrak{N}} N_{v} & \leqq\left.\left(\sum_{v} N_{v}^{2}\right)^{1 / 2} \cdot|\mathfrak{N}|\right|^{1 / 2} \leqq \frac{\varepsilon^{2}}{C(\varepsilon)} \sum_{v} N_{v}^{2}+\frac{C(\varepsilon)}{\varepsilon^{2}}|\mathfrak{N}| \\
& \leqq \frac{\varepsilon^{2}}{C(\varepsilon)} \sum_{v} N_{v}^{2}+C^{\prime}(\varepsilon)|J| .
\end{aligned}
$$

Putting this into (3.17), we find that $\sum_{y \in J\left|x_{j}-y\right|<1 / 2}\left|x_{j}-y\right|^{-1} \leqq-\varepsilon^{2} \Delta+\varepsilon^{2} \sum_{v} N_{v}^{2}+$ $C^{\prime \prime}(\varepsilon)|J|$. Hence,

$$
\sum_{y \in J} \sum_{\left|x_{j}-y\right|<1 / 2}\left|x_{j}-y\right|^{-1} \leqq C \varepsilon^{2}\left(H+C N+C N^{\prime}\right)+C(\varepsilon)|J|
$$

by virtue of (3.3) and (3.10).

Next we record some trivial estimates on partition functions. Let $\Omega_{*}=\Omega(x, R)$ with $\Omega \in \Gamma$, and suppose we place the nucleii at a subset of $Z^{3} \cap \Omega_{*}$. The corresponding $N$-electron Hamiltonian satisfies $H_{N} \geqq \frac{1}{2} H_{N, \Omega_{*}}^{0}-C N-C\left|\Omega_{*}\right|$ by (3.3). Therefore, we have an upper bound

$$
\frac{1}{\left|\Omega_{*}\right|} \ln \sum_{N} e^{\mu N} \operatorname{Tr} e^{-\beta H_{N}} \leqq C(\mu, \beta)
$$

simply because there is a corresponding upper bound for $H_{N}^{0}$. Also, it is obvious that

$$
\frac{1}{\left|\Omega_{*}\right|} \ln \sum_{N} e^{\mu N} \operatorname{Tr} e^{-\beta H_{N}} \geqq-C(\mu, \beta),
$$


simply because there exists $N<C|\Omega|$ and $\psi_{0} \in L_{N}^{2}\left(\Omega_{*}\right)$ with $\left\langle H \psi_{0}, \psi_{0}\right\rangle \leqq C N$. In fact, we can write $\psi_{0}\left(x_{1} \ldots x_{N}\right)$ as an antisymmetrized product of one-electron wave functions, each describing a spherically symmetric electron cloud of radius $1 / 4$ about each nucleus of distance $>1 / 4$ from $\partial \Omega_{*}$.

From (3.19), (3.20) and convexity, we get

$$
\left|\frac{\partial}{\partial \mu} F(\mu, \beta, x, R, \Omega)\right|, \quad\left|\frac{\partial}{\partial \beta} F(\mu, \beta, x, R, \Omega)\right| \leqq C(\mu, \beta, \Omega) \text { for } \Omega \in \Gamma .
$$

The next lemma is a special application of (3.3) and Lemma 3. We fix $D \in \Gamma_{0}$, $\Omega \in \Gamma$, and numbers $R \gg 1, \sigma \ll 1$. Suppose $\theta$ is a function on $R^{3}$ satisfying $\theta(x)=1$ in $D(0, R-\sigma), \theta(x)=0$ outside $D(0, R),|\nabla \theta| \leqq C \sigma^{-1}$ everywhere. Define a kernel

$$
S(x)=\frac{|x|^{-1}}{|D(0, R)|} \int_{D(0, R)} \theta(x+y)(\theta(y)-1) d y .
$$

Place nucleii $\omega_{k}$ at the lattice points of $\Omega_{*}=\Omega\left(x_{*}, R_{*}\right)$. Then we have Lemma 5. $\left|\sum_{j, l} S\left(x_{j}-\omega_{l}\right)-\sum_{l^{\prime} \neq l} S\left(\omega_{l^{\prime}}-\omega_{l}\right)\right| \leqq C \sigma\left(H_{N, \Omega_{*}}+C N+C\left|\Omega_{*}\right|\right)$ on $L_{N}^{2}\left(\Omega_{*}\right)$, if $R_{*}>C R$.

Proof. One computes that

$$
\begin{gathered}
|S(x)| \leqq \frac{C \sigma}{R|x|} \chi_{|x|<C R}, \\
|\nabla S(x)| \leqq \frac{C \sigma}{R|x|^{2}} \chi_{|x|<C R} .
\end{gathered}
$$

Take functions $\phi_{0}, \phi_{1}, \eta$ on $R^{3}$ with $\phi_{0}+\phi_{1}=1, \phi_{0}$ supported in $|x|<1 / 4, \phi_{1}$ supported in $|x|>1 / 8, \int \eta=1, \eta \geqq 0, \eta \in C_{0}^{\infty}\left(|x| \leqq 10^{-3}\right)$. Then write

$$
S=\phi_{0} S+\eta *\left(\phi_{1} S\right)+\left[\phi_{1} S-\eta *\left(\phi_{1} S\right)\right] \equiv S_{1}+S_{2}+S_{3} \text {. }
$$

Estimates (3.22), (3.23) imply

$$
\left|S_{3}(x)\right| \leqq \max _{|y-x|<10^{-3}}\left|\nabla\left(\phi_{1} S\right)(y)\right| \leqq \frac{C \sigma}{R|x|^{2}} \chi_{1 / 10<|x|<C R}
$$

and

$$
\left|\nabla\left(\phi_{1} S\right)(x)\right| \leqq \frac{C \sigma}{R|x|^{2}} \chi_{1 / 10<|x|<C R}
$$

Now

$\sum_{j, l}\left|S_{1}\left(x_{j}-\omega_{l}\right)\right| \leqq(C \sigma / R) \sum_{\left|x_{j}-\omega_{l}\right|<1 / 4}\left|x_{j}-\omega_{l}\right|^{-1} \leqq(C \sigma / R)\left(H+C N+C\left|\Omega_{*}\right|\right)$ by while $\sum_{l^{\prime} \neq l} S_{1}\left(\omega_{l^{\prime}}-\omega_{l}\right)=0$. Hence

$$
\left|\sum_{j, l} S_{1}\left(x_{j}-\omega_{l}\right)-\sum_{l^{\prime} \neq l} S_{1}\left(\omega_{l^{\prime}}-\omega_{l}\right)\right| \leqq \frac{C \sigma}{R}\left(H_{N, \Omega_{\star}}+C N+C\left|\Omega_{*}\right|\right)
$$


Next observe that

$$
\sum_{j, l} S_{2}\left(x_{j}-\omega_{l}\right)-\sum_{l^{\prime} \neq l} S_{2}\left(\omega_{l^{\prime}}-\omega_{l}\right)=\sum_{j} \eta * \mathscr{S}\left(x_{j}\right)-\sum_{l^{\prime}} \eta * \mathscr{S}\left(\omega_{l^{\prime}}\right)
$$

with $\mathscr{S}(y)=\sum_{l}\left(\phi_{1} S\right)\left(y-\omega_{l}\right)$; we have $|\nabla \mathscr{S}|<C \sigma$ by (3.25), and $\mathscr{S}$ is supported in the double of $\Omega_{*}$ since $R_{*}>C R$. Hence, Lemma 3 yields

$$
\left|\sum_{j, l} S_{2}\left(x_{j}-\omega_{l}\right)-\sum_{l^{\prime} \neq l} S_{2}\left(\omega_{l^{\prime}}-\omega_{l}\right)\right|^{2} \leqq C \sigma^{2}\left|\Omega_{*}\right|\left(H+C N+C\left|\Omega_{*}\right|\right),
$$

and therefore

$$
\begin{gathered}
\left|\sum_{j, l} S_{2}\left(x_{j}-\omega_{l}\right)-\sum_{l^{\prime} \neq l} S_{2}\left(\omega_{l^{\prime}}-\omega_{l}\right)\right| \leqq C \sigma\left|\Omega_{*}\right|+\sigma^{-1}\left|\Omega_{*}\right|^{-1} . \\
{\left[\left|\sum_{j, l} S_{2}\left(x_{j}-\omega_{l}\right)-\sum_{l^{\prime} \neq l} S_{2}\left(\omega_{l^{\prime}}-\omega_{l}\right)\right|^{2}\right] \leqq C^{\prime} \sigma\left(H+C^{\prime} N+C^{\prime}\left|\Omega_{*}\right|\right) .}
\end{gathered}
$$

Finally, (3.24) shows that $\sum_{l}\left|S_{3}\left(y-\omega_{l}\right)\right| \leqq C \sigma$ for any $y \in R^{3}$, so that trivially,

$$
\left|\sum_{j, l} S_{3}\left(x_{j}-\omega_{l}\right)-\sum_{l^{\prime} \neq l} S_{3}\left(\omega_{l^{\prime}}-\omega_{l}\right)\right| \leqq C \sigma\left(N+C\left|\Omega_{*}\right|\right) .
$$

Since $S=S_{1}+S_{2}+S_{3}$, Lemma 5 follows from (3.26), (3.27), (3.28).

\section{A Swiss Cheese}

Fix $D \in \Gamma_{0}$. To prove Lemma 1 , we shall partition $R^{3}$ into $D\left(x_{k \alpha}, R_{k}\right)$ and a small residual part. See Lebowitz-Lieb [1], where such a "Swiss cheese" decomposition is used to get existence of the thermodynamic limit.

Lemma 6. Let $1<R_{1}<R_{2}<\cdots<R_{M}$ be radii with $R_{k+1}>2 R_{k}$. Then any cube $Q_{M}^{+}$of side greater than $C M R_{M}$ may be decomposed into a disjoint union $Q^{+}=$ $\bigcup_{k=1}^{M} \bigcup_{\alpha} B_{k \alpha} \cup \bigcup_{\alpha} Q_{\alpha}$ with the following properties.

Each $B_{k \alpha}$ has the form $B_{k \alpha}=D\left(x_{k \alpha}, R_{k}\right)$.

Each $Q_{\alpha}$ is contained in a cube of side 1 .

$\sum_{\alpha}\left|B_{k \alpha}\right| \leqq \frac{10}{M}\left|Q^{+}\right|$for each $k$.

The number of $Q_{\alpha}$ 's is at most $\frac{C}{M}\left|Q^{+}\right|$.

Proof. We give an inductive procedure to construct successively the $B_{M \alpha}, B_{M-1 \alpha}$, $B_{M-2 \alpha}$, etc. We continue applying the procedure until it is impossible to continue, at which time we cut the remaining part of $Q^{+}$into $Q_{\alpha}$ 's.

Assuming we have already constructed the $B_{k \alpha}$ for all $k>j$, our inductive procedure is as follows. (Note that for $j=M$, the inductive hypothesis is fulfilled 
vacuously.) First cut $Q^{+}$into a grid of cubes $\left\{Q_{v}\right\}$ of side $\sim C_{1} R_{j}$. Here $C_{1}$ is a constant chosen so that $D\left(x_{v}, 2 R_{j}\right) \subseteq Q$ when $x_{v}=$ centre of $Q_{v}$. Let $J=\left\{v \mid Q_{v}\right.$ meets none of the $B_{k \alpha}$ already constructed $\}$; at the centre of each $Q_{v}$ we place $B_{v}=D\left(x_{v}, R_{j}\right)$.

Case 1. If $\sum_{v \in J}\left|B_{v}\right|>10 / M\left|Q^{+}\right|$, then since each $B_{v}$ has volume $<\left|Q^{+}\right| / M$, one can pick a subset $\left\{B_{j \alpha}\right\} \cong\left\{B_{v}\right\}_{v \in J}$ so that

$$
\frac{9}{M}\left|Q^{+}\right|<\sum_{\alpha}\left|B_{j \alpha}\right|<\frac{10}{M}\left|Q^{+}\right| .
$$

Note that (4.1), (4.3) hold for $k=j$ if they held for $k>j$. Our inductive step is complete.

Case 2. If $\sum_{v \in J}\left|B_{v}\right| \leqq 10 / M\left|Q^{+}\right|$, then we cut up $Q^{+}$into a grid of unit cubes $\left\{Q_{v}^{0}\right\}$, and define $\left\{Q_{\alpha}\right\}$ to consist of the non-empty intersections of the $Q_{v}^{0}$ with $Q^{+} \backslash \bigcup_{k \alpha} B_{k \alpha}$. The construction of $B_{k \alpha}$ 's and $Q_{\alpha}$ 's is complete.

The first inequality in (4.5) shows that Case 2 must occur for some $j \geqq 1$. When it does occur, we note that the number of $Q_{v}^{0}$ which meet a fixed $\partial B_{k \alpha}$ is at most $C\left|B_{k \alpha}\right| / R_{k}$. Hence the total number of $Q_{v}^{0}$ which meet any of the $\partial B_{k \alpha}$ is at most $\sum_{k} C R_{k}^{-1}\left(\sum_{\alpha}\left|B_{k \alpha}\right|\right) \leqq\left(C\left|Q^{+}\right| / M\right) \cdot \sum_{k} R_{k}^{-1} \leqq C^{\prime} / M\left|Q^{+}\right|$; here we used (4.3).

Similarly, for a fixed $B_{k \alpha}$, the total volume of all the $Q_{v}$ that meet $\partial B_{k \alpha}$ is at most $C R_{j} / R_{k} \cdot\left|B_{k \alpha}\right| ;$ hence the total volume of all the $Q_{v}$ that meet any $\partial B_{k \alpha}$ is at most

$$
\sum_{k>j} \frac{C R_{j}}{R_{k}} \cdot \sum_{\alpha}\left|B_{k \alpha}\right| \leqq \frac{C^{\prime}\left|Q^{+}\right|}{M} \sum_{k>j} R_{j} / R_{k} \leqq \frac{C^{\prime \prime}\left|Q^{+}\right|}{M} .
$$

Also, since we are in Case 2, the total volume of the $Q_{v}$ which meet no $B_{k \alpha}$ is at most $C / M\left|Q^{+}\right|$. Consequently; $\left|Q^{+} \backslash \bigcup_{k \alpha} B_{k \alpha}\right|<C / M\left|Q^{+}\right|$. So the total number of $Q_{v}^{0}$ disjoint from all $B_{k \alpha}$ is at most $C / M\left|Q^{+}\right|$.

Since each $Q_{\alpha}$ is of the form $Q_{v}^{0} \backslash \bigcup_{k \alpha} B_{k \alpha}$ with $Q_{v}^{0}$ either disjoint from all $B_{k \alpha}$ or meeting some $\partial B_{k \alpha}$, property (4.4) is proved. The other properties, (4.1),(4.2), (4.3) are obvious from the construction.

Lemma 6 induces a decomposition of all $R^{3}$ into $B_{k \alpha}$ 's and $Q_{\alpha}$ 's. We just cut $R^{3}$ into congruent subcubes $\left\{Q_{v}^{+}\right\}$of side $\sim 2 C M R_{M}$, and cut each of the $Q_{v}^{+}$via Lemma 6. Thus, $R^{3}=\bigcup_{k \alpha} B_{k \alpha} \cup \bigcup_{\alpha} Q_{\alpha}$. We can assume the decompositions of the different $Q_{v}^{+}$are all translates of one another. Also, we take the $\left\{Q_{v}^{+}\right\}$to have their vertices at lattice points.

Next we introduce a partition of unity $1=\sum_{k \alpha} \theta_{k \alpha}^{2}+\sum_{\alpha} \theta_{\alpha}^{2}$ corresponding to the $B_{k \alpha}$ and $Q_{\alpha}$. More precisely, with $\sigma=M^{-1 / 3}$, we define functions $\theta_{k}, \theta_{k \alpha}, \theta_{\alpha}$ on $R^{3}$ so that

$$
\theta_{k \alpha}(x)=\theta_{k}\left(x-x_{k \alpha}\right) \text {, where } B_{k \alpha}=D\left(x_{k \alpha}, R_{k}\right) .
$$

$\theta_{k}$ is constant on $\partial D(0, r)$ for each $r$, and $\theta_{k}$ is supported in $D\left(0, R_{k}\right)$. 


$$
\begin{aligned}
\left|\partial^{\gamma} \theta_{k}\right| & \leqq C_{\gamma} \sigma^{-|\gamma|} . \\
\operatorname{Supp} \theta_{\alpha} \subseteq \widetilde{Q}_{\alpha} & =\left\{x \mid \operatorname{dist}\left(x, Q_{\alpha}\right)<\sigma\right\} . \\
\left|\partial^{\gamma} \theta_{\alpha}\right| & \leqq C_{\gamma} \sigma^{-|\alpha|} . \\
\sum_{k \alpha} \theta_{k \alpha}^{2}+\sum_{\alpha} \theta_{\alpha}^{2} & =1 .
\end{aligned}
$$

It is easy to define these; first construct $\theta_{k}$ so that $(4.7),(4.8)$ hold and $\left(1-\theta_{k}^{2}\right)^{1 / 2}$ satisfies estimates analogous to (4.8); next define $\theta_{k \alpha}$ by (4.6); and finally construct the $\theta_{\alpha}$.

Note that $\theta_{k \alpha}(x)=1$ for $x \in B_{k \alpha}, \operatorname{dist}\left(x, \partial B_{k \alpha}\right)>\sigma$.

For a vector $\tau \in R^{3}$, we can obviously translate the $B_{k \alpha}, Q_{\alpha}, \theta_{k \alpha}, \theta_{\alpha}$ by $\tau$. Later on, it will be important to do this and then average our estimates over all $\tau \in Q^{+}=$one of the fundamental cubes $\left\{Q_{v}^{+}\right\}$. In particular, we shall need the following identities.

$$
\begin{aligned}
\operatorname{Av}_{\tau \in Q^{+}} \sum_{B_{k \alpha}} \theta_{k \alpha}^{2}(x-\tau) \theta_{k \alpha}^{2}\left(x^{\prime}-\tau\right) & =\sum_{k} \lambda_{k}\left(\theta_{k}^{2} * \tilde{\theta}_{k}^{2}\right)\left(x-x^{\prime}\right), \\
\operatorname{Av}_{\tau \in Q^{+}} \sum_{B_{k \alpha}} \theta_{k \alpha}^{2}(x-\tau) \chi_{B_{k \alpha}}\left(y^{\prime}-\tau\right) & =\sum_{k} \lambda_{k}\left(\theta_{k}^{2} * \tilde{\chi}_{D\left(0, R_{k}\right)}\right)(x-y), \\
\operatorname{Av}_{\tau \in Q^{+}} \sum_{B_{k \alpha}} \chi_{B_{k \alpha}}(y-\tau) \chi_{B_{k \alpha}}\left(y^{\prime}-\tau\right) & =\sum_{k} \lambda_{k}\left(\chi_{D\left(0, R_{k}\right)} * \tilde{\chi}_{D\left(0, R_{k}\right)}\right)\left(y-y^{\prime}\right),
\end{aligned}
$$

where

$$
\lambda_{k}=\left|Q^{+}\right|^{-1} \cdot\left[\text { Number of } x_{k \alpha} \in Q^{+}\right]
$$

and $\tilde{\theta}(x) \equiv \theta(-x)$ for any function $\theta$ on $R^{3}$.

To prove (4.12), (4.13), (4.14), let $B^{0}$ be the set of $x_{k \alpha}$ in $Q^{+}$, and fix a lattice $\Lambda^{+}$in $R^{3}$ so that the fundamental cubes $Q_{v}^{+}$are precisely the translates of $Q^{+}$by vectors in $\Lambda^{+}$. Note that each $x_{k \alpha}$ can be written uniquely as $x_{k \alpha} \equiv x_{k \alpha^{\prime}}+\omega$ with $x_{k \alpha^{\prime}} \in B^{0}$ and $\omega \in \Lambda^{+}$. Now we can write

$$
\begin{aligned}
\mathrm{Av}_{\tau \in Q^{+}} \sum_{B_{k \alpha}} \theta_{k \alpha}^{2}(x-\tau) \theta_{k \alpha}^{2}\left(x^{\prime}-\tau\right) \\
\quad=\left|Q^{+}\right|^{-1} \int_{\tau \in Q^{+}} \sum_{k \alpha} \theta_{k}^{2}\left(x-\tau-x_{k \alpha}\right) \theta_{k}^{2}\left(x^{\prime}-\tau-x_{k \alpha}\right) d \tau \\
=\left|Q^{+}\right|^{-1} \sum_{x_{k \alpha^{\prime}} \sum_{\mathscr{B}^{0}}} \sum_{\omega \in \Lambda^{+}} \int_{\tau \in \mathcal{Q}^{+}} \theta_{k}^{2}\left(x-\tau-\omega-x_{k \alpha^{\prime}}\right) \theta_{k}^{2}\left(x^{\prime}-\tau-\omega-x_{k \alpha^{\prime}}\right) d \tau \\
\quad=\left|Q^{+}\right|^{-1} \sum_{x_{k \alpha^{\prime}} \in \mathscr{P}^{0}} \int_{\xi \in \mathbb{R}^{3}} \theta_{k}^{2}(x-\xi) \theta_{k}^{2}\left(x^{\prime}-\xi\right) d \xi
\end{aligned}
$$

(write $\xi=x_{k \alpha^{\prime}}+\tau+\omega$ with $\tau \in Q^{+}, \omega \in \Lambda^{+}$). This proves (4.12). The proofs of (4.13) and (4.14) are similar.

Finally, with

$$
\begin{gathered}
\bar{\lambda}_{k}=\lambda_{k}\left|D\left(0, R_{k}\right)\right|, \quad \text { we obtain, } \\
0 \leqq \bar{\lambda}_{k} \leqq \frac{10}{M}, \\
1 \geqq \sum_{k=1}^{M} \lambda_{k} \geqq 1-\frac{C}{M}, \quad \text { by (4.3), (4.4), (4.15). }
\end{gathered}
$$


In terms of the $\bar{\lambda}_{k}$, identities (4.12), (4.13), (4.14) become

$$
\begin{aligned}
& \operatorname{Av}_{\tau \in Q^{+}} \sum_{B_{k \alpha}} \theta_{k \alpha}^{2}(x-\tau) \theta_{k \alpha}^{2}\left(x^{\prime}-\tau\right)=\sum_{k} \bar{\lambda}_{k} \frac{\theta_{k}^{2} * \tilde{\theta}_{k}^{2}}{|D| R_{k}^{3}}\left(x-x^{\prime}\right), \\
& \operatorname{Av}_{\tau \in Q^{+}} \sum_{B_{k \alpha}} \chi_{B_{k \alpha}}(x-\tau) \chi_{B_{k \alpha}}\left(x^{\prime}-\tau\right)=\sum_{k} \lambda_{k} \frac{\theta_{k}^{2} * \tilde{\chi}_{D\left(0, R_{k}\right)}(x-y)}{|D| R_{k}^{3}} \\
& \operatorname{Av}_{\tau \in Q^{+}} \sum_{B_{k \alpha}} \chi_{B_{k \alpha}}(y-\tau) \chi_{B_{k \alpha}}\left(y^{\prime}-\tau\right)=\sum_{k} \lambda_{k} \frac{\chi_{D\left(0, R_{k}\right)} * \tilde{\chi}_{D\left(0, R_{k}\right)}\left(y-y^{\prime}\right)}{|D| R_{k}^{3}}
\end{aligned}
$$

\section{An Exploded System}

Fix a cube $Q^{+}=$one of the $Q_{v}^{+}$from the last section, and let $\Omega_{*}=\Omega\left(x_{*}, R_{*}\right)$ with $\Omega \in \Gamma$ and $R_{*} \geqq C M^{2} R_{M}$. Let $\mathscr{B}=\left\{B=B_{k \alpha}\right.$ or $\widetilde{Q}_{\alpha} \mid B+\tau$ meets $\Omega_{*}$ for some $\left.\tau \in Q^{+}\right\}$. To each $B \in \mathscr{B}$ we can associate a vector $\xi_{B} \in Z^{3}$ so that the translates $B+\xi_{B}(B \in \mathscr{B})$ are pairwise disjoint. The $\xi_{B}$ may grow large, but we do not care.

Now for each fixed $\tau \in Q^{+}$we define a simplified statistical mechanics problem, in which Coulomb interactions between the translates $B+\tau(B \in \mathscr{B})$ are turned off. More precisely, set $\widehat{B}=B+\xi_{B}+\tau$ for $B \in \mathscr{B}$, and define the exploded set $\Omega_{\mathrm{ex}}=\bigcup_{B \in \mathscr{B}} \hat{B}$. Define

$$
K_{\mathrm{ex}}(y, z)=\left(\begin{array}{ll}
|y-z|^{-1} & \text { if } y, z \in \widehat{B}_{k \alpha} \text { for some } B_{k \alpha} \in \mathscr{B} \\
0 & \text { otherwise. }
\end{array}\right)
$$

In particular, $K_{\mathrm{ex}}(y, z)=0$ if $y$ and $z$ belong to different components of $\Omega_{\mathrm{ex}}$, or if $y, z$ both belong to $\widehat{B}$ with $B=\widetilde{Q}_{\alpha}$.

Next we place nucleii in $\Omega_{\text {ex }}$. In each $\widehat{B}_{k \alpha} \subset \Omega_{\text {ex }}$ we place a nucleus at each lattice point $\omega \in \widehat{B}_{k \alpha} \cap\left(\Omega_{*}+\xi_{B_{k \alpha}}\right)$. In the $\widetilde{Q}_{\alpha}$ we place no nucleii. Note that $\widetilde{B}_{k \alpha}$ has a nucleus at each lattice point, unless $B_{k \alpha}+\tau$ intersects the complement of $\Omega_{*}$. Let $\hat{y}_{1} \ldots \hat{y}_{N}$, be the nucleii in $\Omega_{\mathrm{ex}}$.

Now for $N$ electrons $x_{1} \ldots x_{N} \in \Omega_{\mathrm{ex}}$ we define a Hamiltonian $H_{N}^{\mathrm{ex}}$ on $L_{N}^{2}\left(\Omega_{\mathrm{ex}}\right)$ by setting

$$
H_{N}^{\mathrm{ex}}=-\Delta_{x}+\sum_{j<k} K_{\mathrm{ex}}\left(x_{j}, x_{k}\right)+\sum_{j<k} K_{\mathrm{ex}}\left(\hat{y}_{j}, \hat{y}_{k}\right)-\sum_{j, k} K_{\mathrm{ex}}\left(x_{j}, \hat{y}_{k}\right)
$$

with Dirichlet boundary conditions. Note that the above constructions are not isomorphic for different $\tau$, because nucleii were placed at lattice points of $B_{k \alpha}+\xi_{B_{k \alpha}}+\tau$.

Since the different components of $\Omega_{\text {ex }}$ act independently in $H_{N}^{\text {ex }}$, we have for the partition functions

$$
\sum_{N} e^{\mu N} \operatorname{Tr} e^{-\beta H_{N}^{\mathrm{ex}}}=\prod_{B \in \mathscr{A}}\left(\sum_{N} e^{\mu N} \operatorname{Tr} e^{-\beta h_{N, B}}\right)
$$

for suitable Hamiltonians $h_{N, B}$ acting on $L_{N}^{2}(\widehat{B})$. If $B=B_{k \alpha} \in \mathscr{B}$ and $(\mathscr{B}+\tau) \cap$ ${ }^{c} \Omega_{*}=\emptyset$, then $h_{N, B}$ is isomorphic to $H_{N, B+\tau}$, so that $\ln \left(\sum_{N} e^{\mu N} \operatorname{Tr} e^{-\beta h_{N, B}}\right)=$ 
$\left|B_{k \alpha}\right| \cdot F\left(\mu, \beta, x_{k \alpha}+\tau, R_{k}, D\right), \quad$ since $\quad B_{k \alpha}=D\left(x_{k \alpha}, R_{k}\right) . \quad$ If $\quad B=B_{k \alpha} \in \mathscr{B}, \quad$ but $(B+\tau) \cap^{c} \Omega_{*} \neq \emptyset$, then in any event $\left|\ln \left(\sum_{N} e^{\mu N} \operatorname{Tr} e^{-\beta h_{N, B}}\right)\right| \leqq C\left|B_{k}\right| \quad$ by (3.19), (3.20). Note also that $\left|F\left(\mu, \beta, x_{k \alpha}+\tau, R_{k}, D\right)\right| \leqq C$, again by (3.19), (3.20). Finally, if $B=\widetilde{Q}_{\alpha}$, then $h_{N, B}$ is the Hamiltonian for $N$ free particles in $B$. Since $B$ is contained in a cube of side 2 , we have $\ln \left(\sum_{N} e^{\mu N} \operatorname{Tr} e^{-\beta h_{N, B}}\right) \leqq C$ for $B=\widetilde{Q}_{\alpha}$, since the partition function is monotone in the domain. Putting these remarks into (5.1), we find that

$$
\begin{aligned}
& \ln \left(\sum_{N} e^{\mu N} \operatorname{Tr} e^{-\beta H_{N}^{\text {ex }}}\right)=\sum_{B_{k \alpha} \in \mathscr{B}}\left|B_{k \alpha}\right| F\left(\mu, \beta, x_{k \alpha}+\tau, R_{k}, D\right) \\
& +\sum_{B_{k \alpha} \in \mathscr{G} ;\left(B_{k \alpha}+\tau\right) \cap{ }^{c} \Omega_{*} \neq \emptyset}\left\{\ln \left(\sum_{N} e^{\mu N} \operatorname{Tr} e^{-h_{N, B}}\right)\right. \\
& \left.-\left|B_{k \alpha}\right| F\left(\mu, \beta, x_{k \alpha}+\tau, R_{k}, D\right)\right\} \\
& +\sum_{B=\widetilde{Q}_{k \alpha} \in \mathscr{B}} \ln \left(\sum_{N} e^{\mu N} \operatorname{Tr} e^{-\beta h_{N, B}}\right) \\
& \leqq \sum_{B_{k \epsilon} \in \mathscr{Q}}\left|B_{k \alpha}\right| \cdot F\left(\mu, \beta, x_{k \alpha}+\tau, R_{k}, D\right) \\
& +C \sum_{B_{k \alpha} \in \mathscr{B}^{\prime}}\left|B_{k \alpha}\right|+C \cdot\left[\text { Number of } \widetilde{Q}_{\alpha} \in \mathscr{B}\right] \text {, } \\
& \text { where } \mathscr{B}^{\prime}=\left\{B_{k \alpha} \in \mathscr{B} \mid\left(B_{k \alpha}+\tau\right) \cap{ }^{c} \boldsymbol{\Omega}_{*} \neq \emptyset\right\} \text {. }
\end{aligned}
$$

Now each $B_{k \alpha} \in \mathscr{B}^{\prime}$ is contained in $E=\left\{x \mid \operatorname{dist}\left(x+\tau, \partial \Omega_{*}\right)<2 \operatorname{diam} Q^{+}\right\}$, since $B_{k \alpha} \cong$ some $Q_{v}^{+}$so that $\operatorname{diam} B_{k \alpha} \leqq \operatorname{diam} Q_{v}^{+}$. Since $\operatorname{diam} Q^{+} \sim C M R_{M}$, while $\Omega_{*}=\Omega\left(x_{*}, R_{*}\right)$ with $R_{*}>C M^{2} R_{M}$, it follows that $E$ has volume $<(C / M)\left|\Omega_{*}\right|$. Hence, $\sum_{B_{B} \in \mathscr{Q}^{\prime}}\left|B_{k \alpha}\right|<(C / M)\left|\Omega_{*}\right|$. Also, the number of $\widetilde{Q}_{\alpha} \in \mathscr{B}$ is at most $(C / M) \mid \Omega_{*}^{\left.B_{\alpha} \in \mathscr{B}\right\}^{\prime}}$ by virtue of (4.4). Hence we can rewrite (5.2) in the form

$$
\ln \left(\sum_{N} e^{\mu N} \operatorname{Tr} e^{-\beta H_{N}^{\text {ex }}}\right) \leqq \sum_{B_{k \alpha} \in \mathscr{Q}}\left|B_{k \alpha}\right| F\left(\mu, \beta, x_{k \alpha}+\tau, R_{k}, D\right)+\frac{C}{M}\left|\Omega_{*}\right| .
$$

Recall that the left-hand side depends on $\tau$.

\section{An Injection of Hilbert Spaces}

We hope to exploit (5.3) by comparing the partition functions for $H_{N}^{\text {ex }}$ and $H_{N, \Omega}$. Since these Hamiltonians live on different Hilbert spaces, it is natural to inject $L_{*}^{2}\left(\Omega_{*}\right)$ into $L_{*}^{2}\left(\Omega_{\mathrm{ex}}\right)$ by an isometry $i$ and then quote the following remark.

Lemma 7. Let $i: E_{1} \rightarrow E_{2}$ be an isometric injection of Hilbert spaces, and let $H_{2}$ be a self-adjoint operator on $E_{2}$. Define $\mathrm{H}_{1}=i^{*} \mathrm{H}_{2} i$ on $E_{i}$. Then $\operatorname{Tr} e^{-\beta \mathrm{H}_{1}} \leqq$ $\operatorname{Tr} e^{-\beta H_{2}}$. 
The proof is immediate by minimax.

Note that $L_{N}^{2}\left(\Omega_{*}\right)$ would be isomorphic to $L_{N}^{2}\left(\Omega_{\mathrm{ex}}\right)$, were it not for the slight overlaps of the $\widetilde{Q}_{\alpha} \in \mathscr{B}$ with the $B_{k \alpha} \in \mathscr{B}$ and one another. This section is just a careful discussion of the technicalities arising from the overlaps.

To prepare for the definition of $i$, we introduce more notation, namely

$$
\theta_{B}=\left(\begin{array}{ll}
\theta_{k \alpha} & \text { if } B=B_{k \alpha} \\
\theta_{\alpha} & \text { if } B=\widetilde{Q}_{\alpha}
\end{array}\right)
$$

Thus, $\theta_{B}$ is supported in $B$, and $\sum_{B \in \mathscr{B}} \theta_{B}^{2}(x-\tau)=1$ for $x \in \Omega_{*}$. Now let $\psi\left(x_{1} \ldots x_{N}\right) \in L_{N}^{2}\left(\Omega_{*}\right)$. We shall define $i \psi$ as a function in $L_{N}^{2}\left(\Omega_{\mathrm{ex}}\right)$. To do so, we must specify the value of $i \psi$ at a point $\left(y_{1} \ldots y_{N}\right) \in \Omega_{\mathrm{ex}}^{N}$. Each $y_{k}$ belongs to a single $\hat{B}_{k}$, so we can write $y_{k}=x_{k}+\xi_{B_{k}}$ with $x_{k} \in B_{k}+\tau$. We define

$$
\left(i_{N} \psi\right)\left(y_{1} \ldots y_{N}\right)=\left(\prod_{k=1}^{N} \theta_{B_{k}}\left(x_{k}-\tau\right)\right) \cdot \psi\left(x_{1} \ldots x_{N}\right) .
$$

Here one interprets $\psi\left(x_{1} \ldots x_{N}\right)=0$ if any of $x_{1} \ldots x_{N}$ lie outside of $\Omega$.

One checks easily that $i_{N}$ injects $L_{N}^{2}\left(\Omega_{*}\right)$ isometrically into $L_{N}^{2}\left(\Omega_{\mathrm{ex}}\right)$. In particular, $i_{N} \psi$ is antisymmetric if $\psi$ is antisymmetric. Also if $\psi$ has one derivative in $L^{2}\left(\Omega_{*}^{N}\right)$ and vanishes on $\partial\left(\Omega_{*}^{N}\right)$, then $i_{N} \psi$ will have one derivative in $L^{2}\left(\Omega_{\mathrm{ex}}^{N}\right)$ and vanish on $\partial\left(\Omega_{\mathrm{ex}}^{N}\right)$. This is because of the factors $\theta_{B} \in C_{0}^{\infty}(B)$ in $i_{N} \psi$. So $i_{N}$ preserves Dirichlet boundary conditions. Define $i$ as the direct sum of the $i_{N}, N \geqq 0$.

Lemma 7 and (5.3) now yield

$$
\ln \left(\sum_{N} e^{\mu N} \operatorname{Tr} e^{-\beta h_{N, \tau}}\right) \leqq \sum_{B_{k \alpha} \in \mathscr{B}}\left|B_{k \alpha}\right| \cdot F\left(\mu, \beta, x_{k \alpha}+\tau, R_{k}, D\right)+\frac{C}{M}\left|\Omega_{*}\right|
$$

for an auxiliary Hamiltonian $h_{N, \tau}=i_{N}^{*} H_{N}^{\text {ex }} i_{N}$ defined on $L_{N}^{2}\left(\Omega_{*}\right)$.

\section{Calculation of the Auxiliary Hamiltonian}

First we recall the definition of $H_{N}^{\mathrm{ex}}$. We have

$$
H_{N}^{\mathrm{ex}}=\sum_{B \in \mathscr{B}} h_{N, B}, \quad \text { where } h_{N, B} \text { acting on } \phi\left(y_{1} \ldots y_{N}\right)
$$

is given by

$$
\begin{aligned}
h_{N, B}= & -\sum_{k} \chi_{\hat{B}}\left(y_{k}\right) \Delta_{y_{k}}+\sum_{j<k}\left|y_{j}-y_{k}\right|^{-1} \chi_{\hat{B}}\left(y_{j}\right) \chi_{\hat{B}}\left(y_{k}\right) \\
& +\sum_{j<k}\left|\hat{y}_{j}-\hat{y}_{k}\right|^{-1} \chi_{\hat{B}}\left(\hat{y}_{j}\right) \chi_{\hat{B}}\left(\hat{y}_{k}\right) \\
& -\sum_{j, k}\left|y_{j}-\hat{y}_{k}\right|^{-1} \chi_{\hat{B}}\left(y_{j}\right) \chi_{\hat{B}}\left(\hat{y}_{k}\right), \quad B=B_{k \alpha} \in \mathscr{B}, \\
h_{N, B}= & -\sum_{k} \chi_{\hat{B}}\left(y_{k}\right) \Delta_{y_{k}}, \quad B=\widetilde{Q}_{\alpha} \in \mathscr{B} .
\end{aligned}
$$

Recall that $\hat{y}_{1} \ldots \hat{y}_{N}$, are the nucleii in $\Omega_{\mathrm{ex}}$. The $h_{N, B}$ of (7.2), (7.3) are essentially the same as $h_{N, B}$ in (5.1). 
By (7.1), we have

$$
\begin{aligned}
\left\langle h_{N, \tau} \psi, \psi\right\rangle & =\left\langle H_{N}^{\mathrm{ex}} i \psi, i \psi\right\rangle=\sum_{B_{1} \ldots B_{N} \in \mathscr{B}}\left\langle H_{N}^{\mathrm{ex}} i \psi, i \psi\right\rangle_{L^{2}\left(\hat{B}_{1} \times \cdots \times \hat{B}_{N}\right)} \\
& =\sum_{B, B_{1} \ldots B_{N} \in \mathscr{B}}\left\langle h_{N, B} i \psi, i \psi\right\rangle_{L^{2}\left(\hat{B}_{1} \times \cdots \times \hat{B}_{N}\right)}
\end{aligned}
$$

On $\hat{B}_{1} \times \cdots \times \widehat{B}_{N}$ we apply (7.2), (7.3) to $\phi\left(y_{1} \ldots y_{N}\right)=i \psi\left(y_{1} \ldots y_{N}\right)=$ $\left(\prod_{l=1}^{N} \theta_{B_{l}}\left(x_{l}-\tau\right)\right) \cdot \psi\left(x_{1} \ldots x_{N}\right)$ with $y_{l}=x_{l}+\xi_{B_{l}}$. To evaluate the characteristic functions, note that $\chi_{B}\left(y_{l}\right)=\left(\begin{array}{ll}\chi_{B}\left(x_{l}-\tau\right) & \text { if } B=B_{l} \\ 0 & \text { otherwise }\end{array}\right)$ for $\left(y_{1} \ldots y_{N}\right) \in \widehat{B}_{1} \times \cdots \times \widehat{B}_{N}$. Also, the $\hat{y}_{k} \in \hat{B}$ are precisely the points $\omega+\xi_{B}$ for $\omega \in Z^{3} \cap \Omega_{*} \cap(B+\tau)$, if $B=B_{k \alpha} \in \mathscr{B}$. There are $\hat{y}_{k} \in \widehat{B}$ if $B=\hat{Q}_{\alpha} \in \mathscr{B}$.

Consequently, the result of putting $\phi=i_{N} \psi$ in (7.2), (7.3) and then substituting into (7.4) is as follows

$$
\begin{aligned}
\left\langle h_{N, \tau} \psi, \psi\right\rangle= & \sum_{B_{1} \ldots B_{N} \in \mathscr{B}}\left\|\nabla\left[\prod_{k=1}^{N} \theta_{B_{k}}\left(x_{k}-\tau\right) \cdot \psi\left(x_{1} \ldots x_{N}\right)\right]\right\|_{L^{2}\left(R^{3 N}\right)}^{2} \\
& +\sum_{B=B_{k \alpha}} \sum_{B_{1} \ldots B_{N} \in \mathscr{B}} \int \prod_{l=1}^{N} \theta_{B_{l}}^{2}\left(x_{l}-\tau\right) \cdot \frac{1}{2} \sum_{j \neq j^{\prime}}\left|x_{j}-x_{j^{\prime}}\right|^{-1} \\
& \times \chi_{B_{j}=B} \chi_{B_{j^{\prime}}=B}\left|\psi\left(x_{1} \ldots x_{N}\right)\right|^{2} d x_{1} \cdots d x_{N} \\
& -\sum_{B=B_{k \alpha} B_{1} \ldots B_{N} \in \mathscr{B}} \sum_{\omega \in \Omega_{\star} \cap Z^{3}} \int \prod_{l=1}^{N} \theta_{B_{l}}^{2}\left(x_{l}-\tau\right) \\
& \cdot \sum_{j}\left|x_{j}-\omega\right|^{-1} \chi_{B_{j}=B} \chi_{B+\tau}(\omega)\left|\psi\left(x_{1} \ldots x_{N}\right)\right|^{2} d x_{1} \ldots d x_{N} \\
& +\sum_{B=B_{k x} B_{1} \ldots B_{N} \in \mathscr{B}} \sum_{l=1} \prod_{l=1}^{N} \theta_{B_{l}}^{2}\left(x_{l}-\tau\right) \cdot \frac{1}{2} \sum_{\substack{\omega \neq \omega^{\prime} \\
\omega \omega^{\prime} \in Z^{3} \cap \Omega_{*}}}\left|\omega-\omega^{\prime}\right|^{-1} \\
& \cdot \chi_{B+\tau}(\omega) \chi_{B+\tau}\left(\omega^{\prime}\right)\left|\psi\left(x_{1} \ldots x_{N}\right)\right|^{2} d x_{1} \ldots d x_{N} \\
\equiv & T+V_{1}-V_{2}+V_{3} .
\end{aligned}
$$

Mercifully, the expressions on the right can be greatly simplified. Let us start with the V's.

Taking the sum over $j, j^{\prime}$ to the outside in the definition of $V_{1}$, we can carry out the inner sum over all the $B_{1} \ldots B_{N}$ except $B_{j}, B_{j^{\prime}}$. Recalling that $\sum_{B \in \mathscr{B}} \theta_{B}^{2}(x-\tau)=1$ for $x \in \Omega_{*}$, we obtain

$$
\begin{aligned}
V_{1}= & \frac{1}{2} \sum_{j \neq j^{\prime}} \int \sum_{B_{k \alpha} \in \mathscr{\theta}} \theta_{B_{k \alpha}}^{2}\left(x_{j}-\tau\right) \theta_{B_{k \alpha}}^{2}\left(x_{j^{\prime}}-\tau\right)\left|x_{j}-x_{j^{\prime}}\right|^{-1} \\
& \cdot\left|\psi\left(x_{1} \ldots x_{N}\right)\right|^{2} d x_{1} \ldots d x_{N} \\
= & \left\langle\frac{1}{2} \sum_{j \neq j^{\prime}} K_{e e}^{\tau}\left(x_{j}, x_{j^{\prime}}\right) \psi, \psi\right\rangle, \text { with }
\end{aligned}
$$




$$
K_{e e}^{\tau}\left(x, x^{\prime}\right)=\sum_{B_{k \alpha} \in \mathscr{B}} \theta_{k \alpha}^{2}(x-\tau) \theta_{k \alpha}^{2}\left(x^{\prime}-\tau\right) \cdot\left|x-x^{\prime}\right|^{-1} .
$$

Similarly, in the definition of $V_{2}$, we can take the sum on $j$ and $\omega$ to the outside, and then perform the inner sum over all the $B_{1} \ldots B_{N}$ except $B_{j}$. The result is

$$
\begin{aligned}
V_{2}= & \sum_{j} \sum_{\omega \in Z^{3} \cap \Omega_{\star}} \int \sum_{B_{k \alpha} \in \mathscr{B}} \theta_{B_{k \alpha}}^{2}\left(x_{j}-\tau\right) \chi_{B_{k \alpha}}(\omega-\tau)\left|x_{j}-\omega\right|^{-1} \\
& \cdot\left|\psi\left(x_{1} \ldots x_{N}\right)\right|^{2} d x_{1} \ldots d x_{N} \\
= & \left\langle\sum_{j} \sum_{\omega \in Z^{3} \cap \Omega_{*}} K_{e p}^{\tau}\left(x_{j}, \omega\right) \psi, \psi\right\rangle, \text { with } \\
K_{e p}^{\tau}(x, \omega)= & \sum_{B_{k \alpha} \in \mathscr{B}} \theta_{k \alpha}^{2}(x-\tau) \chi_{B_{k \alpha}}(\omega-\tau) \cdot|x-\omega|^{-1} .
\end{aligned}
$$

The term $V_{3}$ is the simplest of all, since we can immediately carry out the sum over all the $B_{1} \ldots B_{N}$ to obtain

$$
\begin{aligned}
V_{3} & =\left\langle\frac{1}{2} \sum_{\substack{\omega \neq \omega \\
\omega, \omega^{\prime} \in Z^{3} \cap \Omega \star}} K_{p p}^{\tau}\left(\omega, \omega^{\prime}\right) \psi, \psi\right\rangle, \text { with } \\
K_{p p}^{\tau}\left(\omega, \omega^{\prime}\right) & =\sum_{B_{k \alpha} \in \mathscr{B}} \chi_{B_{k \alpha}}(\omega-\tau) \chi_{B_{k \alpha}}\left(\omega^{\prime}-\tau\right) \cdot\left|\omega-\omega^{\prime}\right|^{-1} .
\end{aligned}
$$

Next we simplify $T$, using the elementary identity $\|\nabla(\theta \psi)\|^{2}=\|\theta \nabla \psi\|^{2}-$ $\langle(\theta \Delta \theta) \psi, \psi\rangle$ for functions $\psi \in C^{\prime}\left(R^{3}\right), \theta \in C_{0}^{\infty}\left(R^{3}\right), \theta$ real. The identity follows trivially from integration by parts. Substituting it into the definition of $T$ yields

$$
\begin{aligned}
T= & \sum_{B_{1} \ldots B_{N} \in \mathscr{B}}\left\|\prod_{k=1}^{N} \chi_{B_{k}}\left(x_{k}-\tau\right) \cdot \nabla\left(x_{1} \ldots x_{N}\right)\right\|_{L^{2}\left(R^{3 N}\right)}^{2} \\
& -\sum_{k} \sum_{B_{1} \ldots B_{N} \in \mathscr{B}}\left\langle\prod_{l \neq k} \theta_{B_{l}}^{2}\left(x_{l}-\tau\right) \cdot \theta_{B_{k}} \Delta \theta_{B_{k}}\left(x_{k}-\tau\right) \psi, \psi\right\rangle .
\end{aligned}
$$

In the first term on the right, we can sum over all the $B_{1} \ldots B_{N}$, while in the second term, we can sum over the $B_{l}$ for $l \neq k$. The result is

$$
\begin{aligned}
T & =\|\nabla \psi\|^{2}-\left\langle\sum_{k} G\left(x_{k}-\tau\right) \psi, \psi\right\rangle, \text { with } \\
G & =\sum_{B \in \mathscr{B}} \theta_{B} \Delta \theta_{B} .
\end{aligned}
$$

Now we can substitute (7.6), (7.8), (7.10), (7.12) into (7.5) to obtain

$$
\begin{aligned}
h_{N, \tau}= & -\Delta+V_{N, \tau}, \quad \text { with } \\
V_{N, \tau}= & \frac{1}{2} \sum_{j \neq k} K_{e e}^{\tau}\left(x_{j}, x_{k}\right)+\frac{1}{2} \sum_{j \neq k} K_{p p}^{\tau}\left(\omega_{j}, \omega_{k}\right) \\
& -\sum_{j, k} K_{e p}^{\tau}\left(x_{j}, \omega_{k}\right)-\sum_{i} G\left(x_{j}-\tau\right) .
\end{aligned}
$$

Here the $\omega_{k}$ denote the lattice points in $\Omega_{*}$, and $K_{e e}^{\tau}, K_{e p}^{\tau}, K_{p p}^{\tau}, G$ are given by (7.7), (7.9), (7.11), (7.13). Note that the sums in these formulas can be extended from $B \in \mathscr{B}$ to all $B=B_{k \alpha}$ in the Swiss cheese; for, the new terms all vanish. The operator (7.14) acts on $L_{N}^{2}\left(\Omega_{*}\right)$ with Dirichlet boundary conditions. 


\section{Averaging over Translates}

So far, we know estimate (6.1) for a Hamiltonian $h_{N, \tau}$ which somewhat resembles the desired $H_{N, \Omega_{*}}$ by virtue of (7.14), (7.15). We can improve the resemblance by averaging over $\tau \in Q^{+}$. Since $\ln \operatorname{Tr} e^{-\beta H}$ is a convex function of a self-adjoint operator $H,(6.1)$ implies

$$
\ln \sum_{N} e^{\mu N} \operatorname{Tr} e^{-\beta h_{N}} \leqq \operatorname{Av}_{\tau \in Q^{+}} \sum_{B_{k \alpha} \in \mathscr{O}}\left|B_{k \alpha}\right| F\left(\mu, \beta, x_{k \alpha}+\tau, R_{k}, D\right)+\frac{C}{M}\left|\Omega_{*}\right|,
$$

where $h_{N}=\mathrm{Av}_{\tau \in Q^{+}} h_{N, \tau}$. Since $x \rightarrow F\left(\mu, \beta, x, R_{k}, D\right)$ is periodic with period lattice $Z^{3}$, while $Q^{+}$is a large cube with its vertices at lattice points, the right-hand side of (8.1) may be rewritten as

$$
\sum_{B_{k \alpha} \in \mathscr{Q}}\left|B_{k \alpha}\right| A v_{x \in Q^{0}} F\left(\mu, \beta, x, R_{k}, D\right)+\frac{C}{M}\left|\Omega_{*}\right|, \quad Q^{0}=\text { unit cube. }
$$

From the definition of $\mathscr{B}$ we have

$$
\sum_{B_{k \alpha} \in \mathscr{O}}\left|B_{k \alpha}\right| \leqq \operatorname{vol}\left\{x \in R^{3} \mid \operatorname{dist}\left(x, \Omega_{*}\right)<\operatorname{diam} Q^{+}\right\} \leqq\left(1+\frac{C}{M}\right)\left|\Omega_{*}\right|,
$$

since $\operatorname{diam} Q^{+} \sim C M R_{M}$ while $\Omega_{*}=\Omega\left(x_{*}, R_{*}\right)$ with $R_{*}>C M^{2} R_{M}$. Hence, expression (8.2) is dominated by $\left|\Omega_{*}\right| \cdot(1+C / M) \cdot \max _{1 \leqq k \leqq M}\left[\mathrm{Av}_{x \in Q^{0}} F\left(\mu, \beta, x, R_{k}, D\right)\right]+$ $C / M\left|\Omega_{*}\right|$. Since we already know that $F\left(\mu, \beta, x, R_{k}, D\right) \leqq C$ by (3.19), it follows that

$$
\left|\Omega_{*}\right|^{-1} \ln \sum_{N} e^{\mu N} \operatorname{Tr} e^{-\beta h_{N}} \leqq \max _{1 \leqq k \leqq M}\left[\mathrm{Av}_{x \in Q^{0}} F\left(\mu, \beta, x, R_{k}, D\right)\right]+\frac{C}{M} .
$$

Now

$$
h_{N}=-\Delta+V_{N}, \quad \text { where } V_{N}=\mathrm{Av}_{\tau \in Q^{+}} V_{N, \tau}
$$

with $V_{N, \tau}$ given by (7.15). To compute the $\tau$-averages of the various terms in (7.15), we use (7.7) and (4.19), (7.9) and (4.20); and (7.11) and (4.21). The result is

$$
V_{N}=\frac{1}{2} \sum_{j \neq k} \tilde{K}_{e e}\left(x_{j}-x_{k}\right)+\frac{1}{2} \sum_{j \neq k} \tilde{K}_{p p}\left(\omega_{j}-\omega_{k}\right)-\sum_{j, k} \tilde{K}_{e p}\left(x_{j}-\omega_{k}\right)-\sum_{j} \bar{G}\left(x_{j}\right),
$$

where the $\omega_{k}$ are the lattice points in $\Omega_{*}$, and

$$
\begin{aligned}
\tilde{K}_{e e}(x) & =|x|^{-1} \sum_{k=1}^{M} \lambda_{k} \frac{\theta_{k}^{2} * \tilde{\theta}_{k}^{2}(x)}{R_{k}^{3}|D|} \\
\tilde{K}_{e p}(x) & =|x|^{-1} \sum_{k=1}^{M} \bar{\lambda}_{k} \frac{\theta_{k}^{2} * \tilde{\chi}_{D\left(0, R_{k}\right)}(x)}{R_{k}^{3}|D|} \\
\tilde{K}_{p p}(x) & =|x|^{-1} \sum_{k=1}^{M} \bar{\lambda}_{k} \frac{\chi_{D\left(0, R_{k}\right)} * \tilde{\chi}_{D\left(0, R_{k}\right)}(x)}{R_{k}^{3}|D|} \\
\bar{G}(x) & =\operatorname{Av}_{\tau \in Q+} \sum_{B \in \mathscr{O}} \theta_{B} \Delta \theta_{B}(x-\tau) .
\end{aligned}
$$


Note that (4.19), (4.20), (4.21) apply here because the restriction to $B \in \mathscr{B}$ in (7.7), (7.9), (7.11) is irrelevant. $\bar{G}$ is a constant, but we won't need to check that. Instead it is enough to note that

$$
\begin{array}{ll}
\left|\theta_{B} \Delta \theta_{B}(x)\right| \leqq C \sigma^{-2} \chi_{\operatorname{dist}(x, \partial B)<\sigma} & \text { if } B=B_{k \alpha} \\
\left|\theta_{B} \Delta \theta_{B}(x)\right| \leqq C \sigma^{-2} \chi_{\tilde{Q}_{\alpha}} & \text { if } B=\widetilde{Q}_{\alpha} .
\end{array}
$$

These estimates are immediate from (4.6)-(4.11) and the remarks immediately following. Integrating, we get

$$
\begin{gathered}
\int_{\tau \in Q^{+}} \sum_{B \in \mathscr{B}}\left|\theta_{B} \Delta \theta_{B}(x-\tau)\right| d \tau \leqq C \sigma^{-2} \cdot \sum_{k=1}^{M} \frac{C \sigma}{R_{k}} \sum_{B_{k \alpha} \cap\left(x-Q^{+}\right) \neq \emptyset}\left|B_{k \alpha}\right| \\
+C \sigma^{-2} \cdot \sum_{\tilde{Q}_{\alpha} \cap\left(x-Q^{+}\right) \neq \emptyset}\left|\tilde{Q}_{\alpha}\right| \leqq \frac{C \sigma^{-2}\left|Q^{+}\right|}{M} \text { by Lemma } 6 .
\end{gathered}
$$

Since we took $\sigma=M^{-1 / 3}$, we have

$$
\mid \bar{G}(x) \leqq C M^{-1 / 3} \text { for all } x \in R^{3} .
$$

\section{Proof of Lemma 1}

The idea is to prove

$$
h_{N} \leqq(1+C \varepsilon) H_{N, \Omega_{*}}+C^{\prime} \varepsilon N+C^{\prime} \varepsilon\left|\Omega_{*}\right|
$$

if $R_{1} \ldots R_{M}$ and $R_{*}$ are as in the statement of Lemma 1. Once we have this, (8.3) yields

$$
\begin{aligned}
& \max _{1 \leqq k \leqq N}\left[\operatorname{Av}_{x \in Q^{0}} F\left(\mu, \beta, x, R_{k}, D\right)\right]+\frac{C}{M} \\
& \geqq\left|\Omega_{*}\right|^{-1} \ln \left[e^{-C^{\prime} \beta \varepsilon\left|\Omega_{*}\right|} \sum_{N} e^{\left(\mu-C^{\prime} \beta \varepsilon\right) N} \operatorname{Tr} e^{-\beta(1+C \varepsilon) H_{N, \Omega_{*}}}\right] \\
& =-C^{\prime} \beta \varepsilon+F\left(\mu-C^{\prime} \beta \varepsilon, \beta(1+C \varepsilon), x_{*}, R_{*}, \Omega\right) .
\end{aligned}
$$

The right hand side is $\geqq F\left(\mu, \beta, x_{*}, R_{*}, \Omega\right)-C^{\prime \prime} \varepsilon$ by (3.21). Here, $C^{\prime \prime}$ depends on $\mu, \beta, \Omega, D$ but not on $x_{*}, R_{*}, R_{1} \ldots R_{M}$ or $\varepsilon$. Thus (9.2) implies

$$
\max _{1 \leqq k \leqq M}\left[\mathrm{Av}_{x \in Q^{0}} F\left(\mu, \beta, x, R_{k}, D\right)\right]+\left(\frac{C}{M}+C^{\prime \prime} \varepsilon\right) \geqq F\left(\mu, \beta, x_{*}, R_{*}, \Omega\right) .
$$

Since $M>C \varepsilon^{-10}$, Lemma 1 follows easily. So our problem is to prove (9.1).

Formulas $(8.4),(8.5),(8.10)$ reduce $(9.1)$ to the estimate

$$
W_{1} \leqq C \varepsilon H_{N, \Omega_{*}}+C^{\prime} \varepsilon N+C^{\prime} \varepsilon\left|\Omega_{*}\right|
$$

with

$$
W_{1}=\frac{1}{2} \sum_{j \neq k} K_{e e}\left(x_{j}-x_{k}\right)+\frac{1}{2} \sum_{j \neq k} K_{p p}\left(\omega_{j}-\omega_{k}\right)-\sum_{j, k} K_{e p}\left(x_{j}-\omega_{k}\right),
$$




$$
\begin{gathered}
K_{e e}(x)=-|x|^{-1}+|x|^{-1} \sum_{k=1}^{M} \bar{\lambda}_{k} \frac{\theta_{k}^{2} * \tilde{\theta}_{k}^{2}(x)}{R_{k}^{3}|D|}, \\
K_{e p}(x)=-|x|^{-1}+|x|^{-1} \sum_{k=1}^{M} \bar{\lambda}_{k} \frac{\theta_{k}^{2} * \tilde{\chi}_{D\left(0, R_{k}\right)}(x)}{R_{k}^{3}|D|} \\
K_{p p}(x)=-|x|^{-1}+|x|^{-1} \sum_{k=1}^{M} \bar{\lambda}_{k} \frac{\chi_{D\left(0, R_{k}\right)} * \tilde{\chi}_{D\left(0, R_{k}\right)}(x)}{R_{k}^{3}|D|} .
\end{gathered}
$$

To prove (9.3), we first correct $W_{1}$ so that electron-electron, electron-proton, and proton-proton all interact with the same potential. We write

$$
\begin{aligned}
& W_{1}=W_{2}+W_{3}+W_{4} \text { with } \\
& W_{2}=V\left[K_{e e}\right], \\
& W_{3}=\sum_{j, k}\left(K_{e e}-K_{e p}\right)\left(x_{j}-\omega_{k}\right)-\sum_{j \neq k}\left(K_{e e}-K_{e p}\right)\left(\omega_{j}-\omega_{k}\right), \\
& W_{4}=\sum_{j \neq k}\left(\frac{1}{2} K_{e e}+\frac{1}{2} K_{p p}-K_{e p}\right)\left(\omega_{j}-\omega_{k}\right) .
\end{aligned}
$$

Now

$$
\left(K_{e e}-K_{e p}\right)(x)=|x|^{-1} \sum_{k=1}^{M} \bar{\lambda}_{k}\left(\theta_{k}^{2 *}\left[\tilde{\theta}_{k}^{2}-\tilde{\chi}_{D\left(0, R_{k}\right)}\right](x) / R_{k}^{3}|D|\right) .
$$

Since $\sum_{k} \bar{\lambda}_{k} \leqq 1$, we can prove that

$$
W_{3} \leqq C \sigma\left(H_{N, \Omega_{*}}+C N+C\left|\Omega_{*}\right|\right),
$$

simply by quoting Lemma 5 with $\theta=\theta_{k}^{2}, R=R_{k}$ and summing against $\bar{\lambda}_{k}$. Also,

$$
\left(\frac{1}{2} K_{e e}+\frac{1}{2} K_{p p}-K_{e p}\right)(x)=\frac{1}{2} \sum_{k=1}^{M} \lambda_{k} \frac{\left[\theta_{k}^{2}-\chi_{D\left(0, R_{k}\right)}\right] *\left[\tilde{\theta}_{k}^{2}-\tilde{\chi}_{D\left(0, R_{k}\right)}\right]}{|D| R_{k}^{2}} \cdot|x|^{-1}
$$

$+[$ Odd function of $x]$.

The odd function cancels when substituted into $\sum_{j \neq k}\left(\frac{1}{2} K_{e e}+\frac{1}{2} K_{p p}-K_{e p}\right)$ $\left(\omega_{j}-\omega_{k}\right)$. One checks that

$$
\left|\frac{\left[\theta_{k}^{2}-\chi_{D\left(0, R_{k}\right)}\right] *\left[\tilde{\theta}_{k}^{2}-\tilde{\chi}_{D\left(0, R_{k}\right)}\right]}{R_{k}^{3}|D|}\right| \leqq \frac{C \sigma^{2}}{R_{k}|x|} \chi_{|x|<C R_{k}} .
$$

(Just use $\left|\widetilde{\theta}_{k}^{2}-\tilde{\chi}_{D\left(0, R_{k}\right)}\right| \leqq \tilde{\chi}_{D\left(0, R_{k}\right) \backslash D\left(0, R_{k}-\sigma\right)}$.) Multiplying this by $\bar{\lambda}_{k} /|x|$, setting $x=\omega_{l}-\omega_{l^{\prime}}$, and summing over all $k=1, \ldots, M$ and all $l \neq l^{\prime}$, we obtain

$$
\left|W_{4}\right| \leqq C \sigma^{2}\left|\Omega_{*}\right|
$$

Recalling that $\sigma=M^{-1 / 3}$ and $M>C \varepsilon^{-10}$, we see that (9.8), (9.9), (9.12) and (9.13) reduce $(9.3)$ to

$$
V\left[K_{e e}\right] \leqq C \varepsilon H_{N, \Omega_{*}}+C^{\prime} \varepsilon N+C^{\prime} \varepsilon\left|\Omega_{*}\right|
$$


Let $\phi_{k} \in C_{0}^{\infty}\left(|x| \leqq R_{k} / 10\right)$ be a radially symmetric approximate identity of total integral 1, satisfying natural estimates. Then with $\rho(x)=\sum_{l} \phi_{k}\left(x-\omega_{l}\right)-$ $\sum_{j} \phi_{k}\left(x-x_{j}\right)$, we have

$V\left[\phi_{k} * \phi_{k} *|x|^{-1}\right]=\frac{1}{2} \int \frac{\rho(x) \rho(y)}{|x-y|}-\left[\phi_{k} * \phi_{k} *|x|^{-1}(0)\right]$

·(TOTAL NUMBER OF ELECTRONS AND NUCLEII)

$$
\geqq-\frac{C}{R_{k}}\left(N+\left|\Omega_{*}\right|\right)
$$

since the double integral is positive. Setting $m_{k}=\left(\theta_{k}^{2} * \widetilde{\theta}_{k}^{2}(0)\right) / R_{k}^{3}|D|$, and recalling that $R_{1}>C \varepsilon^{-10}$, we conclude that

$$
\begin{aligned}
V\left[K_{e e}\right] \leqq & C \varepsilon N+C \varepsilon\left|\Omega_{*}\right|+V\left[K_{e e}+\sum_{k=1}^{M} \bar{\lambda}_{k} m_{k} \phi_{k} * \phi_{k} *|x|^{-1}\right] \\
= & C \varepsilon N+C \varepsilon\left|\Omega_{*}\right|+\left(\sum_{k=1}^{M} \bar{\lambda}_{k} m_{k}-1\right) V\left[|x|^{-1}\right] \\
& +V\left[\sum_{k=1}^{M} \bar{\lambda}_{k}\left\{m_{k} \phi_{k} * \phi_{k} *|x|^{-1}+|x|^{-1} \frac{\theta_{k}^{2} * \tilde{\theta}_{k}^{2}}{R_{k}^{3}|D|}-m_{k}|x|^{-1}\right\}\right] .
\end{aligned}
$$

Now (4.18) and the obvious estimate $1 \geqq m_{k} \geqq 1-C \sigma / R_{k}$ show that $-\varepsilon<$ $\left(\sum_{k=1}^{M} \bar{\lambda}_{k} m_{k}-1\right)<0$, while Lemma 4 shows that $V\left[-|x|^{-1}\right] \leqq C H_{N, \Omega_{*}}+$ $C^{\prime} N+C^{\prime}\left|\Omega_{*}\right|$. So $(9.15)$ yields

with

$$
V\left[K_{e e}\right] \leqq C \varepsilon\left(H_{N, \Omega_{*}}+C^{\prime \prime} N+C^{\prime \prime}\left|\Omega_{*}\right|\right)+V\left[\sum_{k=1}^{M} \lambda_{k} H_{k}\right],
$$

$$
H_{k}(x)=m_{k} \phi_{k} * \phi_{k} *|x|^{-1}+|x|^{-1} \frac{\theta_{k}^{2} * \widetilde{\theta}_{k}^{2}}{R_{k}^{3}|D|}-m_{k}|x|^{-1} \text {. }
$$

Elementary computation shows that

$$
\left|\partial^{\alpha} H_{k}(x)\right| \leqq \frac{C}{R_{k}|x|^{|\alpha|}} \text { if }|\alpha| \leqq 2, \quad \text { or if }|\alpha|=3
$$

and

$$
x \notin\left[D^{*}\left(0, R_{k}\right) \backslash D^{*}\left(0, R_{k}-\sigma\right)\right] .
$$

Here $D^{*}$ is the convex set $\{x-y \mid x, y \in D\} \in \Gamma_{0}$. Also,

$$
H_{k}(x)=0 \quad \text { outside } \quad D^{*}\left(0, R_{k}\right) .
$$

Since $0 \leqq \bar{\lambda}_{k} \leqq C / M$, we see from (9.18), (9.19) that $K=M \cdot \sum_{k=1}^{M} \bar{\lambda}_{k} H_{k}$ satisfies the hypotheses of Lemma 4 , with $D^{*}$ in place of $D$. Hence, Lemma 4 yields 


$$
V\left[\sum_{k=1}^{M} \bar{\lambda}_{k} H_{k}\right] \leqq \frac{C}{M}\left(H_{N, \Omega_{*}}+C^{\prime} N+C^{\prime}\left|\Omega_{*}\right|\right) .
$$

Recalling that $M>C \varepsilon^{-10}$ and substituting (9.20) into (9.16), we obtain the desired estimate (9.14). The proof of Lemma 1 was already reduced to $(9.14)$.

\section{Proof of Lemma 2}

In this section, $C$ denotes a constant independent of $D, \Omega ; C(\Omega)$ denotes a constant independent of $D$; and $C(\Omega, D)$ denotes a constant depending on both $D$ and $\Omega$.

Let $\hat{\omega}_{1} \ldots \hat{\omega}_{L}$ be the lattice points in $\Omega_{*} \backslash D_{*}$. We pick points $\hat{y}_{1} \ldots \hat{y}_{L}$ according to the following procedure.

(a) If $\operatorname{dist}\left(\hat{\omega}_{l}, \partial \Omega_{*}\right)<\frac{1}{10}$, then pick $\hat{y}_{l} \in \Omega_{*}$ to satisfy $\left|\hat{y}_{l}-\hat{\omega}_{l}\right|<\frac{1}{10}$, $\operatorname{dist}\left(\hat{y}_{l}, \partial \Omega_{*}\right)>c(\Omega)$. We can easily construct such a $\hat{y}_{l}$ by taking a convex combination of $\hat{\omega}_{l}$ with a point $y^{0} \in \Omega_{*}$ of maximal distance to $\partial \Omega_{*}$.

(b) If $\operatorname{dist}\left(\hat{\omega}_{l}, \partial D_{*}\right)<\frac{1}{10}$, then pick $\hat{y}_{l} \in \Omega_{*} \backslash D_{*}$ to satisfy $\left|\hat{y}_{l}-\hat{\omega}_{l}\right|>\frac{1}{10}$, $\operatorname{dist}\left(\hat{y}_{l}, \partial D_{*}\right)>\frac{1}{20}$. This is easily done when $R^{\prime}$ is sufficiently large, because then $\partial D_{*}$ will look almost flat around $\hat{\omega}_{l}$.

(c) If $\operatorname{dist}\left(\hat{\omega}_{l}, \partial D_{*}\right)$ and $\operatorname{dist}\left(\hat{\omega}_{l}, \partial \Omega_{*}\right) \geqq \frac{1}{10}$, then set $\hat{y}_{l}=\hat{\omega}_{l}$.

Note that the cases (a), (b), (c) are disjoint since dist $\left(\partial D_{*}, \partial \Omega_{*}\right) \geqq 10$. In all cases we have $\hat{y}_{l} \in \Omega_{*} \backslash D_{*}, \quad\left|\hat{y}_{l}-\hat{\omega}_{l}\right|<\frac{1}{10}, \quad \operatorname{dist}\left(\hat{y}_{l}, \partial D_{*}\right)>\frac{1}{20}$, $\operatorname{dist}\left(\hat{y}_{l}, \partial \Omega_{*}\right)>c(\Omega)$. Since balls of radius $c(\Omega)$ about the $y_{l}$ are pairwise disjoint and contained in $\Omega_{*} \backslash D_{*}$, the number $L$ of $\hat{\omega}_{l}$ is at most $C(\Omega) \varepsilon^{10}\left|\Omega_{*}\right|$. Now fix a spherically symmetric $\phi_{0} \in C_{0}^{\infty}(|x| \leqq c(\Omega))$ with $\left\|\phi_{0}\right\|_{L^{2}}=1$ and $\left\|\nabla \phi_{0}\right\|_{L^{2}}<C(\Omega)$. Form an $L$-electron wave function

$$
\psi_{0}\left(x_{1} \ldots x_{L}\right)=\frac{1}{\sqrt{L !}} \sum_{\pi}(\operatorname{sgn} \pi) \prod_{l=1}^{L} \phi_{0}\left(x_{\pi(l)}-\hat{y}_{l}\right),
$$

where $\pi$ runs over permutations of $1 \ldots L$. We check easily that

$$
\psi_{0} \in C_{0}^{\infty}\left(\left[\Omega_{*} \backslash D_{*}\right]^{L}\right), \quad\left\|\psi_{0}\right\|=1, \quad\left\langle H_{L, \Omega * \backslash D_{*}} \psi_{0}, \psi_{0}\right\rangle<C(\Omega) \varepsilon^{10}\left|\Omega_{*}\right| .
$$

Now define an isometric injection $i_{N}: L_{N}^{2}\left(D_{*}\right) \rightarrow L_{N+L}^{2}\left(\Omega_{*}\right)$ by

$$
\begin{aligned}
\left(i_{N} \psi\right)\left(x_{1} \ldots x_{N+L}\right)= & \frac{1}{\sqrt{(N+L) !}} \sum_{\pi}(\operatorname{sgn} \pi) \psi\left(x_{\pi(1)} \ldots x_{\pi(N)}\right) \\
& \cdot \psi_{0}\left(x_{\pi(N+1)} \ldots x_{\pi(N+L)}\right)
\end{aligned}
$$

where $\pi$ runs over permutations of $1 \ldots N+L$.

For $\|\psi\|=1$, one compute that

$$
\begin{aligned}
\left\langle H_{N+L, \Omega_{\star}} i_{N} \psi, i_{N} \psi\right\rangle= & \left\langle H_{N, D_{\star}} \psi, \psi\right\rangle+\left\langle H_{L, \Omega_{*} D_{*}} \psi_{0}, \psi_{0}\right\rangle \\
& +\left\langle\left[\sum_{k} F\left(\omega_{k}\right)-\sum_{j} F\left(x_{j}\right)\right] \psi, \psi\right\rangle,
\end{aligned}
$$


where $\omega_{k}$ runs over the lattice points of $D_{*}$, and

$$
F(x)=\sum_{l=1}^{L}\left(\left|x-\hat{\omega}_{l}\right|^{-1}-\left|x-\hat{y}_{l}\right|^{-1}\right) .
$$

The proof of (10.2) uses the mean-value property of $|x|^{-1}$, radial symmetry of $\phi_{0}$, and the fact that $\phi_{0}\left(x-\hat{y}_{l}\right)$ is supported away from $D_{*}$.

Now with $\phi$ as in (3.18), we have

$$
\begin{gathered}
\left|\sum_{k} \phi * \phi * F\left(\omega_{k}\right)-\sum_{j} \phi * \phi * F\left(x_{j}\right)\right| \\
\leqq C R^{2.5}+C R^{-2.5}\left|\sum_{k} \phi * \phi * F\left(\omega_{k}\right)-\sum_{j} \phi * \phi * F\left(x_{j}\right)\right|^{2} \\
\leqq C R^{2.5}+C R^{-2.5}\|\nabla(\eta \cdot[\phi * F])\|_{L^{2}}^{2} \cdot\left(H_{N, D_{*}}+C N+C(\Omega) \cdot\left|\Omega_{*}\right|\right)
\end{gathered}
$$

by Lemma 3 . Here we are using $C(\Omega) \cdot\left|\Omega_{*}\right|$ as an upper bound on the number of nucleii in $D_{*}$; and we take $\eta(x)=1$ if $\operatorname{dist}\left(x, \Omega_{*}\right) \leqq R, \eta(x)=0$ if $\operatorname{dist}\left(x, \Omega_{*}\right) \geqq 2 R$, and $|\nabla \eta| \leqq C(\Omega) R^{-1}$ everywhere. We introduced $\eta$ because Lemma 3 applies to functions of compact support.

The obvious estimates

and

$$
|\phi * F(x)| \leqq \sum_{\hat{y}_{l} \neq \hat{\omega}_{l}}\left(\left|x-\hat{\omega}_{l}\right|+1\right)^{-2} C \leqq C(\Omega, D) \quad \text { for } \operatorname{dist}\left(x, \Omega_{*}\right)>R
$$

$$
|\nabla \phi * F(x)| \leqq \sum_{\hat{y}_{l} \neq \omega_{l}}\left(\left|x-\hat{\omega}_{l}\right|+1\right)^{-3} C \leqq \frac{C(\Omega, D)}{1+\operatorname{dist}\left(x, \partial \Omega_{*} \cup \partial D_{*}\right)} \text { for all } x,
$$

show that

$$
\|\nabla(\eta \cdot[\phi * F])\|_{L^{2}}^{2} \leqq C(\Omega, D) \cdot R^{2}
$$

So (10.4) yields

$$
\begin{aligned}
\left|\sum_{k} \phi * \phi * F\left(\omega_{k}\right)-\sum_{j} \phi * \phi * F\left(x_{j}\right)\right| & \leqq C(\Omega, D) R^{-1 / 2}\left(H_{N, D_{*}}+C N+C(\Omega)\left|\Omega_{*}\right|\right) \\
& <\varepsilon^{10}\left(H_{N, D_{*}}+C N+C(\Omega)\left|\Omega_{*}\right|\right)
\end{aligned}
$$

if $R$ is large enough. On the other hand,

$$
\begin{aligned}
\left|\sum_{k} F\left(\omega_{k}\right)-\sum_{j} F\left(x_{j}\right)\right| \leqq & \left|\sum_{k} \phi * \phi * F\left(\omega_{k}\right)-\sum_{j} \phi * \phi * F\left(x_{j}\right)\right| \\
& +C \sum_{y \in J} \sum_{j}\left|x_{j}-y\right|^{-1} \chi_{\left|x_{j}-y\right|<1 / 3}, J \\
& =\left\{\hat{\omega}_{l} \text { of distance }<1 / 20 \text { from } \partial D_{*}\right\} .
\end{aligned}
$$

To see (10.6), note that the $\omega_{k}$ have distance at least 1 to the $\hat{\omega}_{l}$ and hence also distance at least $9 / 10$ to the $\hat{y}_{l}$, while the $\hat{y}_{l}$ have distance at least $1 / 20$ to the $D_{*}$ and hence also to the $x_{j}$. So if $z=x_{j}$ or $\omega_{k}$ and $z^{\prime}=\hat{y}_{l}$ or $\hat{\omega}_{l}$, then $\left|z-z^{\prime}\right|^{-1}=\left(\phi * \phi *|x|^{-1}\right)\left(z-z^{\prime}\right)$ unless $z=x_{j}, z^{\prime}=\hat{\omega}_{l}$. 
Now estimate (3.8) gives an upper bound $C \varepsilon^{2}\left(H_{N, D_{*}}+C N+C(\Omega)\left|\Omega_{*}\right|\right)+$ $C(\varepsilon) \cdot C(D) R^{2}$ for the last term on the right-hand side of (10.6). For $R$ large enough, $C(\varepsilon) \cdot C(D) R^{2}<\varepsilon^{2}\left|\Omega_{*}\right|$, so that (10.5) and (10.6) yield

$$
\left|\sum_{k} F\left(\omega_{k}\right)-\sum_{j} F\left(x_{j}\right)\right| \leqq C \varepsilon^{2}\left(H_{N, D}+C N+C(\Omega) \cdot\left|\Omega_{*}\right|\right) .
$$

Therefore, by (10.2) and our estimate for the energy of $\psi_{0}$, we know

$$
i_{N}^{*} H_{N+L, \Omega_{*}} i_{N} \leqq\left(1+C(\Omega) \varepsilon^{2}\right) H_{N, D_{*}}+C^{\prime}(\Omega) \varepsilon^{2} N+C^{\prime}(\Omega) \varepsilon^{2}\left|\Omega_{*}\right| .
$$

Using Lemma 7 and (10.7), we can assert

$$
\begin{aligned}
\sum_{N} e^{\bar{\mu} N} \operatorname{Tr} e^{-\bar{\beta} H_{N, \Omega_{*}}} & \geqq \sum_{N} e^{\bar{\mu}(N+L)} \operatorname{Tr} e^{-\bar{\beta} H_{N+L, \Omega_{*}}} \\
& \geqq e^{\bar{\mu} L} \sum_{N} e^{\bar{\mu} N} \operatorname{Tr} e^{-\bar{\beta} i N_{N} H_{N+L, \Omega_{*} i_{N}}} \\
& \geqq e^{\bar{\mu} L-C^{\prime}(\Omega) \varepsilon^{2} \bar{\beta} \mid \Omega_{*}} \sum_{N} e^{\left(\bar{\mu}-C^{\prime}(\Omega) \varepsilon^{2} \bar{\beta}\right) N} \operatorname{Tr} e^{-\bar{\beta}\left(1+C(\Omega) \varepsilon^{2}\right) H_{N, N_{*}}}
\end{aligned}
$$

Pick $\bar{\mu}, \bar{\beta}$ so that $\bar{\beta}\left(1+C(\Omega) \varepsilon^{2}\right)=\beta$ and $\bar{\mu}-C^{\prime}(\Omega) \varepsilon^{2} \bar{\beta}=\mu$. Thus, $|\beta-\bar{\beta}|$, $|\mu-\bar{\mu}| \leqq C^{\prime \prime}(\Omega) \varepsilon^{2}$, and (10.8) yields

$$
\left|\Omega_{*}\right| F\left(\bar{\mu}, \bar{\beta}, \Omega_{*}\right)>\left|D_{*}\right| F\left(\mu, \beta, D_{*}\right)-C^{\prime \prime}(\Omega) \varepsilon^{2}\left|\Omega_{*}\right| .
$$

Estimates (3.21) give $\left|F\left(\mu, \beta, \Omega_{*}\right)-F\left(\bar{\mu}, \bar{\beta}, \Omega_{*}\right)\right| \leqq C(\Omega) \varepsilon^{2}$, and $\left|D_{*}\right|<\left|\Omega_{*}\right|<$ $\left(1+\varepsilon^{10}\right)\left|D_{*}\right|$. Therefore (10.9) implies $F\left(\mu, \beta, \Omega_{*}\right) \geqq F\left(\mu, \beta, D_{*}\right)-C(\Omega) \varepsilon^{2}$, which is stronger than the conclusion of Lemma 2.

Acknowledgments. It is a pleasure to thank Webster Hughes, who contributed an important simplification of our proof; also Elliott Lieb and Barry Simon provided encouragement and helpful discussions. I am grateful to Mo Kirkham for fast, accurate typing.

\section{Reference}

1. Lebowitz, J., Lieb, E.: The Constitution of Matter: Existence of thermodynamics for systems composed of electrons and nucleii, Adv. Math. 9, 316-398 (1972)

Communicated by B. Simon

Received June 29, 1984 
